\title{
Relativistic non-resistive viscous magnetohydrodynamics from the kinetic theory: a relaxation time approach
}

\author{
Ankit Kumar Panda, Ashutosh Dash, Rajesh Biswas and Victor Roy \\ School of Physical Sciences, National Institute of Science Education and Research, HBNI, \\ 752050, Jatni, India \\ E-mail: ankitkumar.panda@niser.ac.in, ashutosh.dash@niser.ac.in, \\ rajeshphysics143@gmail.com, victor@niser.ac.in
}

ABSTRACT: We derive the relativistic non-resistive, viscous second-order magnetohydrodynamic equations for the dissipative quantities using the relaxation time approximation. The Boltzmann equation is solved for a system of particles and antiparticles using ChapmanEnskog like gradient expansion of the single-particle distribution function truncated at second order. In the first order, the transport coefficients are independent of the magnetic field. In the second-order, new transport coefficients that couple magnetic field and the dissipative quantities appear which are different from those obtained in the 14-moment approximation [1] in the presence of a magnetic field. However, in the limit of the weak magnetic field, the form of these equations are identical to the 14-moment approximation albeit with different values of these coefficients. We also derive the anisotropic transport coefficients in the Navier-Stokes limit.

KeYwORDS: Phenomenological Models, Heavy Ion Phenomenology

ARXIV EPRINT: 2011.01606 


\section{Contents}

1 Introduction 1

2 Relativistic magnetohydrodynamics 3

2.1 Equations of motion of the electromagnetic field 3

2.2 Kinetic theory and hydrodynamics 5

2.3 Conservation of energy-momentum tensor of fluid and field $\quad 7$

2.4 Ideal and dissipative non-resistive magnetohydrodynamics 8

3 Formalism and results $\quad 8$

3.1 Boltzmann equation 8

3.2 Expansion in gradients $\quad 9$

$\begin{array}{lll}3.3 & \text { First order equations } & 10\end{array}$

$\begin{array}{ll}3.4 \text { Second order equations } & 11\end{array}$

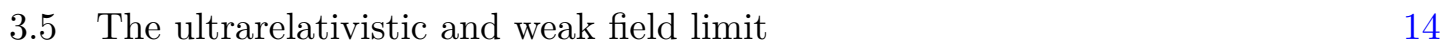

$\begin{array}{ll}3.6 & \text { The Navier-Stokes limit } \\ \end{array}$

$\begin{array}{lll}4 \text { Conclusion } & 17\end{array}$

$\begin{array}{lr}\text { A Thermodynamic integrals } & 18\end{array}$

$\begin{array}{ll}\text { B Second order relaxation equation for dissipative stresses } & 19\end{array}$

$\begin{array}{ll}\text { B.1 Shear stress } & 19\end{array}$

$\begin{array}{ll}\text { B.2 Bulk stress } & 21\end{array}$

B.3 Diffusion current 23

$\begin{array}{ll}\text { C Projection tensors } & 25\end{array}$

D General expressions of transport coefficients $\quad 26$

\section{Introduction}

The ubiquitous magnetic field seems to have played a great role in shaping and working of our present-day universe. We see magnetic fields at the very largest scales in the universe. They are usually very weak, no more than a million times weaker than Earth's magnetic field, but they exist throughout the known universe. Although weak, often the magnetic fields are called the sturdy unsung workhorses of astrophysics and cosmology. On the other hand, one of the strongest steady-state magnetic field in the known universe can be found on the surface of a type of neutron star called Magnetars. Surprisingly, the strongest transient magnetic fields in the universe are manmade and can be found on earth during the initial 
stages of heavy-ion collisions at Relativistic Heavy Ion Collider (RHIC) near Brookhaven, New York and at Large Hadron Collider (LHC) near Geneva, Switzerland. These strongest magnetic fields are produced by fast-moving charged protons (usually having Lorentz factor $\gamma \sim 100$ or larger) inside the colliding nuclei of heavy ions ( $\mathrm{Pb}$ or $\mathrm{Au})$. The strength of the magnetic field produced in such collider experiments for example for a typical peripheral $\mathrm{Au}+\mathrm{Au}$ collisions at $\sqrt{s_{N N}}=200 \mathrm{GeV}$ may reach as high as $\left(\sim 10^{18}-10^{19}\right.$ Gauss $)$ refs. [2$6]$, this is almost three to four orders of magnitude larger than those found on Magnetars. The magnitude of the produced magnetic field is expected to grow linearly with the centerof-mass energy but the lifetime of these strong fields reduces for higher energy collisions. The heavy-ion collisions also produce a new form of very hot and dense matter known as quark-gluon plasma (QGP). The success of relativistic hydrodynamics in describing the space-time evolution of the QGP created in high-energy heavy-ion collisions refs. [7-10] and the existence of very large magnetic fields in these collisions indicates that one should take into account the proper interaction of magnetic fields with QGP. Especially since the QGP and the subsequent hadronic phase are known to be electrically conducting refs. [11-13].

Relativistic magnetohydrodynamics (RMHD) is one of the self-consistent macroscopic frameworks that describes the evolution of mutually interacting charged fluid and electromagnetic fields. In several recent works, the effect of the electromagnetic fields on the QGP fluid in the context of special relativistic systems have been studied in refs. [14-20]. Almost all of them involves numerical solutions of RMHD equations, the analytic solutions for some simplified cases are presented in refs. [21-27]. The transport coefficients such as the shear, bulk viscosity etc. are taken as input to the RMHD simulation, but they are determined from an underlying microscopic theory in refs. [28-36]. It is a well known fact that a straightforward extension of non-relativistic viscous fluid formulations (a.k.a. NavierStokes equation) to the relativistic regime (without magnetic field) refs. [37, 38] leads to unacceptable acuasal and linearly unstable behaviour in refs. [39-41]. These issues were later addressed and resolved by Israel and Stewart (IS) who developed a causal and stable second-order formalism refs. [42, 43]. The order of the theory is determined by the presence of different order terms in the gradient expansion of the hydrodynamic quantities such as fluid four-velocity $u^{\mu}$, temperature $T$, etc. in the energy-momentum tensor. Although IS resolves the major problem, the theory is known to be causal and stable in a restricted manner refs. [44-47]. Recently, there are some new developments in the formulation of first-order theories which is potentially causal and stable refs. [48-54]. However, we note that in the newly developed theory the existence of a relaxation time scale (usually found in the secondorder theories) in the definition of non-equilibrium hydrodynamics variables needs further investigation. Although initially developed as a phenomenological theory, the IS theory was later derived from the underlying kinetic theory using Grad's moment method. One of the limitations of the moment method is the absence of a smallness parameter such as Knudsen number $(\mathrm{Kn})$ which otherwise would have helped to systematically improve the result by keeping higher-order terms. Later, more concrete and updated form of the IS equations (without electromagnetic fields) were derived from the kinetic theory in refs. [55-64].

It was only recently that the second-order causal magnetohydrodynamics equations were derived for non-resistive in ref. [1] and resistive case in ref. [65] for a single compo- 
nent system of spinless particles (no antiparticle) using a 14-moment approximation. In the current work we consider contribution from both particles and antiparticles, henceforth whenever we compare our results with ref. [1] we report the result for particles only. In ref. [66] we showed that this new theory of second-order relativistic MHD is causal and stable under linear perturbation. In this paper, we derive the RMHD equations for the non-resistive case using Chapman-Enskog expansion of the single-particle distribution function within relaxation time approximation (RTA). Here we consider both particles and antiparticles while calculating the relaxation equations for the dissipative quantities. Due to the presence of smallness parameter $\mathrm{Kn}$ in the RTA formalism we have the freedom to construct magnetohydrodynamics equations order by order and calculate corresponding transport coefficients. It is necessary to use a causal theory of magnetohydrodynamics to study other important phenomena associated with strong magnetic fields. For example, the coexistence of the strongest magnetic field in the universe and the hot dense medium of quarks and gluons also opens up possibilities to experimentally verify some of the fundamental issues of Quantum Chromo-Dynamics (QCD). One such fascinating phenomena is "chiral magnetic effect" (CME) where an induced charge current is supposed to be produced parallel to the magnetic field in a chiral imbalance system refs. [67, 68]. Some other important phenomenon associated with strong magnetic fields are chiral separation effect ref. [69], chiral Hall effect ref. [70], chiral vortical effect ref. [71] etc. A chiral kinetic theory framework is currently under development to further explore these important phenomena see refs. [72-74].

The manuscript is organized as follows: in section 2 we give a textbook-like introduction to the energy-momentum tensor for the electromagnetic fields and the fluid-matter, we also discuss the kinetic theory definition of various hydrodynamical variables in the same section. In section 3 we present the first and second order magneto-hydrodynamic equations of motion for the non-resistive dissipative fluid. We conclude this work in section 4 . Throughout the paper we use the natural units, $\hbar=c=k_{B}=\epsilon_{0}=\mu_{0}=1$ and the metric tensor in flat space-time is $g^{\mu \nu}=\operatorname{diag}(+1,-1,-1,-1)$. The time-like fluid four velocity $u^{\mu}$ satisfy $u_{\mu} u^{\mu}=1$. Also, we use the following decomposition for the partial derivative: $\partial_{\mu} \equiv u_{\mu} u_{\nu} \partial^{\nu}+\left(g_{\mu \nu}-u_{\mu} u_{\nu}\right) \partial^{\nu}=u_{\mu} D+\nabla_{\mu}$. The $\nabla^{\alpha} u^{\beta}$ is decomposed as:

$$
\nabla^{\alpha} u^{\beta}=\omega^{\alpha \beta}+\sigma^{\alpha \beta}+\frac{1}{3} \theta \Delta^{\alpha \beta},
$$

where $\omega^{\alpha \beta}=\left(\nabla^{\alpha} u^{\beta}-\nabla^{\beta} u^{\alpha}\right) / 2$ is the anti-symmetric vorticity tensor, $\sigma^{\alpha \beta} \equiv \nabla^{\langle\alpha} u^{\beta\rangle}=$ $\frac{1}{2}\left(\nabla^{\alpha} u^{\beta}+\nabla^{\beta} u^{\alpha}\right)-\frac{1}{3} \theta \Delta^{\alpha \beta}$ is the symmetric-traceless tensor and $\theta \equiv \partial_{\mu} u^{\mu}$ is the expansion scalar. The fourth-rank projection tensor is defined as $\Delta_{\alpha \beta}^{\mu \nu}=\frac{1}{2}\left(\Delta_{\alpha}^{\mu} \Delta_{\beta}^{\nu}+\Delta_{\beta}^{\mu} \Delta_{\alpha}^{\nu}\right)-$ $\frac{1}{3} \Delta^{\mu \nu} \Delta_{\alpha \beta}$.

\section{Relativistic magnetohydrodynamics}

\subsection{Equations of motion of the electromagnetic field}

Here we start by giving some text-book like introduction to the relativistically covariant formulation of electrodynamics. Without any loss of generality the second rank antisym- 
metric electromagnetic field tensor $F^{\mu \nu}$ can be defined in terms of the electric $E^{\mu}$, magnetic field $B^{\mu}$ four-vectors (defined later) and four-velocity $u^{\mu}$ as in refs. [75-77]:

$$
F^{\mu \nu}=E^{\mu} u^{\nu}-E^{\nu} u^{\mu}+\epsilon^{\mu \nu \alpha \beta} u_{\alpha} B_{\beta},
$$

its dual counter-part is given by:

$$
\tilde{F}^{\mu \nu}=B^{\mu} u^{\nu}-B^{\nu} u^{\mu}-\epsilon^{\mu \nu \alpha \beta} u_{\alpha} E_{\beta},
$$

where $E^{\mu}=F^{\mu \nu} u_{\nu}$ and $B^{\mu}=\tilde{F}^{\mu \nu} u_{\nu}=\frac{1}{2} \epsilon^{\mu \nu \alpha \beta} u_{\nu} F_{\alpha \beta}$. Also, using the anti-symmetric property of the $F^{\mu \nu}$ it is easy to see that both $E^{\mu}$ and $B^{\mu}$ are orthogonal to $u^{\mu}$ i.e., $E^{\mu} u_{\mu}=B^{\mu} u_{\mu}=0$. Futhermore notice that in the rest frame $u^{\mu}=(1, \mathbf{0})$ we have $E^{\mu}:=$ $(0, \mathbf{E})$, and $B^{\mu}:=(0, \mathbf{B})$, where $\mathbf{E}, \mathbf{B}$ corresponds to the electric and magnetic field three vectors with $\mathrm{E}^{i}:=F^{i 0}$ and $\mathrm{B}^{i}:=-\frac{1}{2} \epsilon^{i j k} F_{j k}$.

We can write the Maxwell's equations in a covariant form as:

$$
\begin{aligned}
& \partial_{\mu} F^{\mu \nu}=J^{\nu}, \\
& \partial_{\mu} \tilde{F}^{\mu \nu}=0,
\end{aligned}
$$

where $J^{\nu}$ is the electric charge four-current which acts as the source of electromagnetic field. It can be tensor decomposed in a fluid with four velocity $u^{\mu}$ in the following manner:

$$
J^{\mu}=j^{\mu}+d^{\mu},
$$

where $j^{\mu}$ is the conduction current and $d^{\mu}=\Delta_{\nu}^{\mu} J^{\nu}$ is the charge diffusion current with $n_{q}=$ $u_{\mu} J^{\mu}$ the proper net charge density. If we assume a linear constitutive relation between $j^{\mu}$ and $E^{\mu}$ (Ohm's law) then $j^{\mu}=\sigma^{\mu \nu} E_{\nu}$ where $\sigma^{\mu \nu}$ is the conductivity tensor. Also note that by construction $u_{\mu} j^{\mu}=0$ which imply that the conduction current exists even for the vanishing net charge. The solution of eqs. (2.3), (2.4) along with a given $J^{\mu}$ in eq. (2.5) completely specify the electro-magnetic field evolution. $J^{\mu}$ acts as a coupling between the fluid and the fields because it contains the fluid informations such as fluid conductivity $\sigma^{\mu \nu}$, net charge density $n_{q}$ etc., and act as a source in the Maxwell's equations. Incidentally, for a single component gas as considered here the net charge is equivalent to net number density and the following relation holds $n_{q}=q n_{f}$, where $n_{f}$ corresponds to net number density.

We assume here that the fluid under consideration does not possess polarisation or magnetisation and thus the electromagnetic field stress-energy tensor can be written as:

$$
T_{E M}^{\mu \nu}=-F^{\mu \lambda} F_{\lambda}^{\nu}+\frac{1}{4} g^{\mu \nu} F^{\alpha \beta} F_{\alpha \beta} .
$$

Now taking the partial derivative of the field stress-energy tensor we get the equation of motion to be:

$$
\partial_{\mu} T_{E M}^{\mu \nu}=-F^{\nu \lambda} J_{\lambda}
$$

Up until now, no external (electromagnetic field) sources were considered. So, the charge current density comes only from the fluid i.e., $J^{\mu}=J_{f}^{\mu}$. However, in presence of an external source current $J_{\text {ext }}^{\mu}$ (for example, the spectator protons in heavy-ion collisions act like an 
external source for the electromagnetic fields in the QGP) the total current is a combination of conduction and external current densities:

$$
J^{\mu}=J_{f}^{\mu}+J_{e x t}^{\mu}
$$

In this case, the external current density acts as a source term in the energy-momentum conservation equation (discusses later in detail). In this work, we consider an ideal MHD limit which corresponds to very large magnetic Reynolds number $R_{m} \gg 1$. The magnetic Reynolds number is given as, $R_{m}=L U \sigma \mu$, where $L$ is the characteristic length or time scale of the QGP, $U$ is the characteristic velocity of the flow and $\mu$ is the magnetic permeability of QGP. The large $R_{m}$ limit can be attributed to a very large/infinite electrical conductivity. But the induced charge density due to the electromagnetic field $J_{i n d}^{\mu}=\sigma E^{\mu}$ (here $\sigma$ is the isotropic electrical conductivity i.e., $\sigma^{\mu \nu}=\sigma g^{\mu \nu}$ ) has to be finite, so to maintain that $E^{\mu} \rightarrow 0$ for this case. This brings our electromagnetic tensor $F^{\mu \nu}$ to the following form:

$$
F^{\mu \nu} \rightarrow B^{\mu \nu}=\epsilon^{\mu \nu \alpha \beta} u_{\alpha} B_{\beta} .
$$

Using eqs. (2.8) and (2.9) in the Maxwell's equations eq. (2.3) we get:

$$
\epsilon^{\mu \nu \alpha \beta}\left(u_{\alpha} \partial_{\mu} B_{\beta}+B_{\beta} \partial_{\mu} u_{\alpha}\right)=J_{f}^{\nu}+J_{e x t}^{\nu} .
$$

Now writing the energy-momentum tensor for the electromagnetic case by using eqs. (2.6) and (2.9) we get:

$$
T_{E M}^{\mu \nu} \rightarrow T_{B}^{\mu \nu}=\frac{B^{2}}{2}\left(u^{\mu} u^{\nu}-\Delta^{\mu \nu}-2 b^{\mu} b^{\nu}\right),
$$

where $B^{\mu} B_{\mu}=-B^{2}$ and $b^{\mu}=\frac{B^{\mu}}{B}$ with the property $b^{\mu} u_{\mu}=0$ and $b^{\mu} b_{\mu}=-1$. Furthermore from eq. (2.9) one can show that $B^{\mu \nu} B_{\mu \nu}=2 B^{2}$ so we can introduce another anti-symmetric tensor defined as:

$$
b^{\mu \nu}=-\frac{B^{\mu \nu}}{B},
$$

with the following properties: $b^{\mu \nu} u_{\nu}=b^{\mu \nu} b_{\nu}=0$ and $b^{\mu \nu} b_{\mu \nu}=2$.

\subsection{Kinetic theory and hydrodynamics}

In this section, we define a few hydrodynamical variables from the kinetic theory. We start with the equilibrium distribution function for particles given by $f_{0}$ and is defined as:

$$
f_{0}=\frac{1}{e^{\beta(u \cdot p)-\alpha}+r},
$$

where $u \cdot p \equiv u_{\mu} p^{\mu}$ and $r=+1,-1,0$ are for fermions, bosons and Boltzmann gas, respectively. Here $\beta=\frac{1}{T}$ is the inverse temperature, $u^{\mu}$ is the four-velocity, $p^{\mu}$ is the four-momentum and $\alpha=\frac{\mu}{T}$ is the ratio of chemical potential to temperature with $\mu$ being the chemical potential. For antiparticles $\alpha \rightarrow-\alpha$ and $f_{0} \rightarrow \bar{f}_{0}$.

For dissipative fluid, one can define fluid four velocity $u^{\mu}$ in more than one ways but the most popular choices are by Eckart and Landau-Lifshitz. In this work, the fluid four velocity has been defined by the Landau-Lifshitz frame which corresponds to the vanishing heat flux in the local rest frame of the fluid. For that case, the net four current $N^{\mu}$ and the 
energy-momentum tensor $T_{f}^{\mu \nu}$ can be decomposed in terms of $u^{\mu}$, the projector operator $\Delta^{\mu \nu}$, and dissipative fluxes diffusion current $V_{f}^{\mu}$, the shear $\pi^{\mu \nu}$ and bulk stress $\Pi$ in the following way:

$$
\begin{aligned}
N^{\mu} & =n_{f} u^{\mu}+V_{f}^{\mu}, \\
T_{f}^{\mu \nu} & =\epsilon u^{\mu} u^{\nu}-(P+\Pi) \Delta^{\mu \nu}+\pi^{\mu \nu},
\end{aligned}
$$

where $n_{f}$ is the net number density, $\epsilon$ is the energy density, $P$ is the isotropic pressure of fluid. According to kinetic theory framework the energy-momentum tensor and the particle four current of a fluid can be defined in terms of moments of the single particle distribution function $f$ in the following way:

$$
\begin{aligned}
T_{f}^{\mu \nu} & =\int d p p^{\mu} p^{\nu}(f+\bar{f}), \\
N^{\mu} & =\int d p p^{\mu}(f-\bar{f}),
\end{aligned}
$$

where $d p=g d^{3} \mathbf{p} /\left[(2 \pi)^{3} p^{0}\right]$ with $p^{0}=\sqrt{\mathbf{p}^{2}+m^{2}}, m$ being the mass, $g$ is the degeneracy factor. For near-equilibrium system the distribution function can be decomposed into equilibrium $f_{0}$ and a correction to it $\delta f$ as $f=f_{0}+\delta f$ (for antiparticle $\delta f \rightarrow \delta \bar{f}$ ). The explicit form of the $\delta f$ is obtained from the Boltzmann equation and it depend on the scheme used. For example the $\delta f$ for the case of relativistic ideal gas in magnetic field in terms of Grad's fourteen moment method was derived in ref. [1]. With the above definition of $N^{\mu}$ and $T^{\mu \nu}$ all other thermodynamic variables can be defined as:

$$
\begin{aligned}
\epsilon & \equiv u_{\mu} u_{\nu} T^{\mu \nu}=u_{\mu} u_{\nu} \int d p p^{\mu} p^{\nu}\left(f_{0}+\bar{f}_{0}\right), \\
n_{f} & \equiv u_{\mu} N^{\mu}=u_{\mu} \int d p p^{\mu}\left(f_{0}-\bar{f}_{0}\right), \\
P & \equiv-\frac{\Delta_{\mu \nu}}{3} T^{\mu \nu}=-\frac{\Delta_{\mu \nu}}{3} \int d p p^{\mu} p^{\nu}\left(f_{0}+\bar{f}_{0}\right), \\
V_{f}^{\mu} & \equiv \Delta_{\nu}^{\mu} N^{\nu}=\Delta_{\nu}^{\mu} \int d p p^{\nu}(\delta f-\delta \bar{f}), \\
\Pi & \equiv-\frac{\Delta_{\mu \nu}}{3} \delta T^{\mu \nu}=-\frac{\Delta_{\mu \nu}}{3} \int d p p^{\mu} p^{\nu}(\delta f+\delta \bar{f}), \\
\pi^{\mu \nu} & \equiv \Delta_{\alpha \beta}^{\mu \nu} \delta T^{\mu \nu}=\Delta_{\alpha \beta}^{\mu \nu} \int d p p^{\alpha} p^{\beta}(\delta f+\delta \bar{f}),
\end{aligned}
$$

where $\delta T^{\mu \nu}=-\Pi \Delta^{\mu \nu}+\pi^{\mu \nu}$. For a single component fluid the net current $N^{\mu}$ and charged current are related as:

$$
J_{f}^{\mu}=q N^{\mu},
$$

where $q$ is the magnitude of electric charge.

For later use, we express the integrals in eq. (2.18) to eq. (2.20) in terms of thermodynamics integrals $I_{n q}^{(m) \pm}$ (defined in appendix A) as:

$$
\begin{aligned}
\epsilon & =I_{20}^{(0)+}, \\
n_{f} & =I_{10}^{(0)-}, \\
P & =-I_{21}^{(0)+},
\end{aligned}
$$

here \pm corresponds to the addition or subtraction of $\bar{f}$. 


\subsection{Conservation of energy-momentum tensor of fluid and field}

In a simple fluid (for zero magnetic field) the energy-momentum tensor and the particle currents are conserved separately according to the following conservation laws:

$$
\begin{aligned}
\partial_{\mu} N^{\mu} & =0, \\
\partial_{\mu} T_{f}^{\mu \nu} & =0 .
\end{aligned}
$$

Now let us consider a fluid interacting with the electro-magentic field and let $T^{\mu \nu}$ be the total energy-momentum tensor (field+fluid). $T^{\mu \nu}$ can be written as a sum of energymomentum tensor of the fluid and the electromagnetic field as:

$$
T^{\mu \nu}=T_{f}^{\mu \nu}+T_{E M}^{\mu \nu} .
$$

In general, the total energy-momentum tensor contains additional terms in ref. [76] which cannot be unambiguously attributed to either fluid or field but in case of constant susceptibilities and vanishing $E^{\mu}$ these terms vanishes and eq. (2.30) is a good approximation. Note that due to the conservation of electric charges, the charge current of the fluid is individually conserved:

$$
\partial_{\mu} J_{f}^{\mu}=0 .
$$

If we have an external charge current, it will act as a source in the energy momentum conservation equation which in this case takes the following form:

$$
\partial_{\mu} T^{\mu \nu}=-F^{\nu \lambda} J_{e x t, \lambda} .
$$

The conservation equation for electromagnetic field eq. (2.7) with external source takes the following form:

$$
\partial_{\mu} T_{E M}^{\mu \nu}=-F^{\nu \lambda}\left(J_{f, \lambda}+J_{e x t, \lambda}\right) .
$$

Using eq. (2.30) and eqs. (2.32), (2.33) we get:

$$
\partial_{\mu} T_{f}^{\mu \nu}=F^{\nu \lambda} J_{f, \lambda} .
$$

Usually, the total energy-momentum tensor of an isolated system remains conserved but in case of the presence of an external source (here external charge current) the conservation is satisfied only when a proper source term is taken into account. As we can see that in this case, the fluid evolution depends on the fluid charge current through eq. (2.34).

It is convenient to express the conservation equations in an alternative form by taking projection along and perpendicular to fluid four velocity. The parallel projection of eq. (2.33) and eq. (2.34) gives:

$$
\begin{aligned}
u_{\nu} \partial_{\mu} T_{E M}^{\mu \nu} & =0, \\
u_{\nu} \partial_{\mu} T_{f}^{\mu \nu} & =0,
\end{aligned}
$$

it implies that the energy density of the fluid and the field are unaffected by the charge currents/magnetic field. The perpendicular projection of eq. (2.33) and eq. (2.34) using 
eq. (2.8) gives

$$
\begin{aligned}
\Delta_{\nu}^{\alpha} \partial_{\mu} T_{E M}^{\mu \nu} & =B b^{\alpha \lambda}\left(J_{f, \lambda}+J_{e x t, \lambda}\right) \\
\Delta_{\nu}^{\alpha} \partial_{\mu} T_{f}^{\mu \nu} & =-B b^{\alpha \lambda} J_{f, \lambda} .
\end{aligned}
$$

This shows that unlike energy density, the momentum density of the fluid depends on the diffusion current/magnetic field and the momentum density of the field also depends on the external current, along with the fluid diffusion current.

\subsection{Ideal and dissipative non-resistive magnetohydrodynamics}

In case the fluid is ideal, the total energy momentum tensor takes the form:

$$
T_{(0)}^{\mu \nu}=\left(\epsilon+\frac{B^{2}}{2}\right) u^{\mu} u^{\nu}-\left(P+\frac{B^{2}}{2}\right) \Delta^{\mu \nu}-B^{2} b^{\mu} b^{\nu} .
$$

If the fluid is dissipative with finite shear and bulk viscosity, the energy-momentum tensor in that case becomes:

$$
T^{\mu \nu}=\left(\epsilon+\frac{B^{2}}{2}\right) u^{\mu} u^{\nu}-\left(P+\Pi+\frac{B^{2}}{2}\right) \Delta^{\mu \nu}-B^{\mu} B^{\nu}+\pi^{\mu \nu} .
$$

The system of equations is closed with the constitutive relation of charged-current $J_{f}^{\mu}=$ $\boldsymbol{n}_{f} u^{\mu}+d_{f}^{\mu}$ and with an Equation of State (EoS) relating thermodynamic pressure to energy and number density $p=p\left(\epsilon, n_{f}\right)$. Now using eq. (2.31), eq. (2.34) along with eq. (2.14), eq. (2.15) and using the thermodynamic integrals given in eq. (A.7) and eq. (A.8) we get the evolution equations for $\dot{\alpha}, \dot{\beta}$ and $\dot{u}^{\mu}$ which are of the following forms:

$$
\begin{aligned}
\dot{\alpha} & =\frac{1}{D_{20}}\left[-J_{30}^{(0)+}\left(n_{f} \theta+\partial_{\mu} V_{f}^{\mu}\right)+J_{20}^{(0)-}\left\{\left(h n_{f}+\Pi\right) \theta-\pi^{\mu \nu} \sigma_{\mu \nu}\right\}\right], \\
\dot{\beta} & =\frac{1}{D_{20}}\left[-J_{20}^{(0)-}\left(n_{f} \theta+\partial_{\mu} V_{f}^{\mu}\right)+J_{10}^{(0)+}\left\{\left(h n_{f}+\Pi\right) \theta-\pi^{\mu \nu} \sigma_{\mu \nu}\right\}\right], \\
\dot{u}^{\mu} & =\frac{1}{(1+\Pi) h n_{f}}\left[\frac{n_{f}}{\beta}\left(\nabla^{\mu} \alpha-h \nabla^{\mu} \beta\right)-\Delta_{\nu}^{\mu} \partial_{\gamma} \pi^{\gamma \nu}+\nabla^{\mu} \Pi-q B b^{\mu \nu} V_{f, \nu}\right],
\end{aligned}
$$

where $D_{20}=J_{30}^{(0)+} J_{10}^{(0)+}-J_{20}^{(0)-} J_{20}^{(0)-}, h=\frac{\epsilon+P}{n_{f}}$.

\section{$3 \quad$ Formalism and results}

\subsection{Boltzmann equation}

The relativistic Boltzmann equation (RBE) in the presence of a non-zero force $\mathcal{F}^{\nu}$ is given by:

$$
p^{\mu} \partial_{\mu} f+\mathcal{F}^{\nu} \frac{\partial}{\partial p^{\nu}} f=C[f]
$$

where $f(\mathbf{x}, \mathbf{p}, t)$ is the one particle distribution function characterising the phase space density of the particles, $C[f]$ is the collision kernel. In the ideal MHD limit the electric field vanishes in the local rest frame of the fluid, hence the only contribution to the force 
term in the RBE is due to the magnetic field which is $\mathcal{F}^{\nu}:=q F^{\nu \alpha} p_{\alpha}$ for particles where $q$ is the electric charge of the particles, and $F^{\mu \nu}=-B b^{\mu \nu}$ (eq. (2.9)).

Much simplification can be made if we assume the collision kernel of the form relaxation time approximation given by Bhatnagar-Gross-Krook (BGK) for non-relativistic systems and by Anderson-Witting in ref. [78] for the relativistic systems of the following form $C[f]=-\frac{u \cdot p}{\tau_{c}} \delta f$ where $\tau_{c}$ is the relaxation time or the time taken by the particles away from equilibrium to come to the equilibrium state and $\delta f=f-f_{0}$ denotes the deviation from the equilibrium distribution $f_{0}$. Now substituting the collision kernel into the eq. (3.1) we get the RBE for the particles:

$$
p^{\mu} \partial_{\mu} f+q F^{\sigma \nu} p_{\nu} \frac{\partial}{\partial p^{\sigma}} f=-\frac{u \cdot p}{\tau_{c}} \delta f
$$

The corresponding equation for the antiparticles are obtained by replacing $q \rightarrow-q$ and $f \rightarrow \bar{f}$. The above approximation of the collision kernel in the Boltzmann equation has its own limitation e.g., the relaxation time $\left(\tau_{c}\right)$ here does not depend on momentum of the colliding particles as mentioned above. The interaction between colliding particles are such that the mean free path is larger than the interaction length. In other words we assume that $f(\mathbf{x}, \mathbf{p}, t)$ is a smoothly varying function over the duration of collisions and distances of the order of the interaction range.

\subsection{Expansion in gradients}

One can cast eq. (3.2) to the well-known hydrodynamic gradient expansion form in ref. [79], given the system is close to equilibrium, i.e., the collision kernel is almost vanishing, $C[f] \approx$ 0 . In the absence of an electromagnetic field eq. (3.2) can be written in the following form:

$$
\left(\frac{\tau_{c}}{u \cdot p} p^{\mu} \partial_{\mu}+1\right) f=(\mathcal{D}+1) f=f_{0}
$$

where we have introduced the operator $\mathcal{D} \equiv \frac{\tau_{c}}{u \cdot p} p^{\mu} \partial_{\mu}$. Multiplying the inverse operator $(\mathcal{D}+1)^{-1}$ in the above equation and subsequently doing a power series expansion gives

$$
f=\sum_{n=0}^{\infty}(-\mathcal{D})^{n} f_{0}=\sum_{n=0}^{\infty}\left(-\frac{\tau_{c}}{u \cdot p} p^{\mu} \partial_{\mu}\right)^{n} f_{0} .
$$

The above expansion is valid given that $\mathrm{Kn}=\tau_{c} \partial \ll 1$, which of course is also the relevant expansion parameter. If one identifies the typical gradient strength to be proportional to the temperature, $\partial \sim T$, then the expansion parameter is $\tau_{c} T$ and the series expansion is valid for $\tau_{c} T \ll 1$.

However, in the presence of a magnetic field, the naive gradient expansion breaks since one introduces a new scale into the problem which is proportional to the strength of the magnetic field. Defining the operator $\mathcal{D}_{B} \equiv \frac{\tau_{c}}{u \cdot p}\left(p^{\mu} \partial_{\mu}+q F^{\sigma \nu} p_{\nu} \frac{\partial}{\partial p^{\sigma}}\right)$ and doing a similar power series expansion, gives the following result

$$
\begin{aligned}
f & =\sum_{n=0}^{\infty}\left(-\mathcal{D}_{B}\right)^{n} f_{0}, \\
& =\sum_{n=0}^{\infty}\left[-\frac{\tau_{c}}{u \cdot p}\left(p^{\mu} \partial_{\mu}+q F^{\sigma \nu} p_{\nu} \frac{\partial}{\partial p^{\sigma}}\right)\right]^{n} f_{0} .
\end{aligned}
$$


Along with previous assumption $\tau_{c} T \ll 1$, one has to also assume that $\tau_{c} / r_{g} \ll 1$, where $r_{g}=k_{\perp} / q B$ is the gyroradius (Larmor radius) and $k_{\perp}$ is the component of the momentum perpendicular to the direction of the magnetic field. In the plasma, the typical transverse momentum of particle $k_{\perp} \sim T$ and thus one has also to satisfy the condition $\chi=q B \tau_{c} / T \ll 1$.

In the following, $f$ is obtained by keeping the terms up to second order, i.e., $n=2$ in eq. (3.5), which yields

$$
f=f_{0}+\delta f^{(1)}+\delta f^{(2)}
$$

where

$$
\delta f^{(1)}=-\frac{\tau_{c}}{u \cdot p}\left(p^{\mu} \partial_{\mu}+q F^{\sigma \nu} p_{\nu} \frac{\partial}{\partial p^{\sigma}}\right) f_{0}
$$

and

$$
\delta f^{(2)}=\frac{\tau_{c}}{u \cdot p}\left(p^{\mu} \partial_{\mu}+q F^{\sigma \nu} p_{\nu} \frac{\partial}{\partial p^{\sigma}}\right)\left[\frac{\tau_{c}}{u \cdot p}\left(p^{\alpha} \partial_{\alpha}+q F^{\rho \beta} p_{\beta} \frac{\partial}{\partial p^{\rho}}\right) f_{0}\right] .
$$

The above expression can be simplified by using the relations $q B b^{\mu \nu} p_{\nu} \frac{\partial f_{0}}{\partial p^{\mu}}=0$ and $F^{\mu \nu}=$ $-B b^{\mu \nu}$, which gives

$$
f=f_{0}+\delta \tilde{f}^{(1)}+\delta \tilde{f}^{(2)}
$$

where

$$
\delta \tilde{f}^{(1)}=-\frac{\tau_{c}}{u \cdot p} p^{\mu} \partial_{\mu} f_{0}
$$

and

$$
\delta \tilde{f}^{(2)}=\frac{\tau_{c}}{u \cdot p} p^{\mu} \partial_{\mu}\left[\frac{\tau_{c}}{u \cdot p} p^{\alpha} \partial_{\alpha} f_{0}\right]-\frac{\tau_{c}}{u \cdot p} q B b^{\sigma \nu} p_{\nu} \frac{\partial}{\partial p^{\sigma}}\left[\frac{\tau_{c}}{u \cdot p} p^{\alpha} \partial_{\alpha} f_{0}\right] .
$$

Similarly for antiparticles $\delta \bar{f}$ is calculated by replacing $f_{0} \rightarrow \bar{f}_{0}$ and $q \rightarrow-q$. It is important to note that although the magnetic field does not enter explicitly in the first term of $\delta \tilde{f}^{(2)}$, moreover it does enter implicitly through the acceleration term $\dot{u}^{\mu}$, i.e., eq. (2.43) while taking higher-order moments of such terms.

\subsection{First order equations}

The term $\delta \tilde{f}^{(1)}$ neither depends explicitly nor implicitly on the magnetic field, since in the first-order equations we keep terms till order $\mathcal{O}(\partial)$ in eqs. (2.41)-(2.43). However, for completeness, we nevertheless discuss here the result for the first-order terms in gradient expansion. The results of the present section are the same as in ref. [63] which was derived for zero magnetic fields.

We evaluate the dissipative part of the energy-momentum tensor (which includes the shear, bulk viscosity, and diffusion) using $\delta \tilde{f}^{(1)}$ and $\delta \tilde{\bar{f}}^{(1)}$ in the following,

$$
\begin{aligned}
\pi_{(1)}^{\mu \nu} & =\Delta_{\alpha \beta}^{\mu \nu} \int d p p^{\alpha} p^{\beta}\left(\delta \tilde{f}^{(1)}+\delta \tilde{\bar{f}}^{(1)}\right) \\
\Pi_{(1)} & =-\frac{\Delta_{\mu \nu}}{3} \int d p p^{\mu} p^{\nu}\left(\delta \tilde{f}^{(1)}+\delta \tilde{\bar{f}}^{(1)}\right) \\
V_{(1)}^{\mu} & =\Delta_{\alpha}^{\mu} \int d p p^{\alpha}\left(\delta \tilde{f}^{(1)}-\delta \tilde{\bar{f}}^{(1)}\right)
\end{aligned}
$$


Substituting the value of $\delta \tilde{f}^{(1)}$ from eq. (3.10) into the above eqs. (3.11)-(3.13), after some algebra we get the following relations. For shear viscous pressure

$$
\pi_{(1)}^{\mu \nu}=2 \tau_{c} \beta_{\pi} \sigma^{\mu \nu},
$$

where $\beta_{\pi}=\beta J_{42}^{(1)+}$ and $\sigma^{\mu \nu}=\Delta_{\alpha \beta}^{\mu \nu} \nabla^{\alpha} u^{\beta}$.

For the bulk viscous pressure,

$$
\Pi_{(1)}=-\tau_{c} \beta_{\Pi} \theta
$$

where $\theta=\partial_{\mu} u^{\mu}$ and

$$
\beta_{\Pi}=\frac{5 \beta}{3} J_{42}^{(1)+}+\mathcal{X} J_{31}^{(0)+}-\mathcal{Y} J_{21}^{(0)-},
$$

with the terms $\mathcal{X}$ and $\mathcal{Y}$ being

$$
\begin{aligned}
& \mathcal{X}=\frac{J_{10}^{(0)+}(\epsilon+P)-J_{20}^{(0)-} n_{f}}{D_{20}}, \\
& \mathcal{Y}=\frac{J_{20}^{(0)-}(\epsilon+P)-J_{30}^{(0)+} n_{f}}{D_{20}},
\end{aligned}
$$

Finally for the net particle diffusion current,

$$
V_{(1)}^{\mu}=\tau_{c} \beta_{V} \nabla^{\mu} \alpha
$$

where $\beta_{V}=\frac{n_{f}}{\epsilon+P} J_{21}^{(0)-}-J_{21}^{(1)-}$.

\subsection{Second order equations}

We derive the second-order relaxation type equations for the shear, bulk viscous pressure and diffusion current by taking the appropriate moments of $\delta \tilde{f}^{(2)}$. While deriving these equations, we keep terms up to order $\mathcal{O}\left(\partial^{2}\right)$. We know that the second-order transport coefficient differs even for zero magnetic fields when calculated using RTA in ref. [63] and moment method in ref. [60]. We might expect a similar result for non-zero magnetic field as well.

For shear stress. By definition the second order contribution to the shear stress tensor is given by:

$$
\pi_{(2)}^{\mu \nu}=\Delta_{\alpha \beta}^{\mu \nu} \int d p p^{\alpha} p^{\beta}\left(\delta \tilde{f}^{(2)}+\delta \tilde{\tilde{f}}^{(2)}\right),
$$

where $\delta \tilde{f}^{(2)}$ is given in eq. (3.2). Note that the total shear stress is the combination of first and second order terms:

$$
\pi^{\mu \nu}=\pi_{(1)}^{\mu \nu}+\pi_{(2)}^{\mu \nu}
$$

Evaluating the integral of eq. (3.19) (see appendix (B.1) for details) and adding it to the eq. (3.20) we get the evolution equation for the shear stress tensor:

$$
\begin{aligned}
\frac{\pi^{\mu \nu}}{\tau_{c}}= & -\dot{\pi}^{\mu \nu}+2 \beta_{\pi} \sigma^{\mu \nu}+2 \pi_{\gamma}^{\langle\mu} \omega^{\nu\rangle \gamma}-\tau_{\pi \pi} \pi_{\gamma}^{\langle\mu} \sigma^{\nu\rangle \gamma}-\delta_{\pi \pi} \pi^{\mu \nu} \theta+\lambda_{\pi \Pi} \Pi \sigma^{\mu \nu}-\tau_{\pi V} V^{\langle\mu} \dot{u}^{\nu\rangle} \\
& +\lambda_{\pi V} V^{\langle\mu} \nabla^{\nu\rangle} \alpha+l_{\pi V} \nabla^{\langle\mu} V^{\nu\rangle}+\delta_{\pi B} \Delta_{\eta \beta}^{\mu \nu} q B b^{\gamma \eta} g^{\beta \rho} \pi_{\gamma \rho}-\tau_{c} q B \tau_{\pi V B} \dot{u}^{\langle\mu} b^{\nu\rangle \sigma} V_{\sigma} \\
& -\tau_{c} q B \lambda_{\pi V B} V_{\gamma} b^{\gamma\langle\mu} \nabla^{\nu\rangle} \alpha-q \tau_{c} \delta_{\pi V B} \nabla^{\langle\mu}\left(B^{\nu\rangle \gamma} V_{\gamma}\right)
\end{aligned}
$$


the resulting second order transport co-efficients are given in terms of thermodynamic integrals in table 2. Note, that the co-efficients $\tau_{\pi V}$ and $\lambda_{\pi V}$ contain the derivatives of $l_{\pi V}$ ref. [59], while $\tau_{\pi V B}, \lambda_{\pi V B}$ contain derivatives of $\delta_{\pi V B}$, respectively. We notice that the last four terms contain the magnetic field explicitly and are new when compared to the case for zero magnetic field in ref. [63]. Compared to the calculation done for non-zero magnetic field using a 14-moment approximation in ref. [1], we found only the first ten terms on the r.h.s. have a similar form or analogous structure. However, the last three terms are new and do not appear in the 14- moment approximation. We will discuss this issue in section 3.5.

For bulk stress. Similar to the shear viscosity, we derive the second order evolution equation for the bulk viscous stress. By the definition:

$$
\Pi_{(2)}=-\frac{\Delta_{\alpha \beta}}{3} \int d p p^{\alpha} p^{\beta}\left(\delta \tilde{f}^{(2)}+\delta \tilde{\tilde{f}}^{(2)}\right) .
$$

Evaluating the above integral by using $\delta \tilde{f}^{(2)}$ from eq. (3.2) and noting the fact that the total bulk stress is a combination of first and second-order terms i.e., $\Pi=\Pi_{(1)}+\Pi_{(2)}$ after some algebra (the details are given in appendix B.2 we get the evolution equation for bulk stress:

$$
\begin{aligned}
\frac{\Pi}{\tau_{c}}= & -\dot{\Pi}-\delta_{\Pi \Pi} \Pi \theta+\lambda_{\Pi \pi} \pi^{\mu \nu} \sigma_{\mu \nu}-\tau_{\Pi V} V \cdot \dot{u}-\lambda_{\Pi V} V \cdot \nabla \alpha-l_{\Pi V} \partial \cdot V-\beta_{\Pi} \theta \\
& +\tau_{c} \tau_{\Pi V B} \dot{u}_{\alpha} q B b^{\alpha \beta} V_{\beta}-\tau_{c} q \delta_{\Pi V B} \nabla_{\mu}\left(B b^{\mu \beta} V_{\beta}\right)-\tau_{c} q B \lambda_{\Pi V B} b^{\mu \beta} V_{\beta} \nabla_{\mu} \alpha
\end{aligned}
$$

where the second-order transport coefficients are given in terms of the thermodynamic integrals in table 3 and we use eq. (3.17) for the expression of $\mathcal{X}$ and $\mathcal{Y}$. Coefficients $\tau_{\Pi V}, \lambda_{\Pi V}$ contain derivatives of $l_{\Pi V}$, while $\tau_{\Pi V B}, \lambda_{\Pi V B}$ contain derivatives of $\delta_{\Pi V B}$, respectively. The last three terms of the above equation are new compared to that of ref. [63] and are magnetic field dependent. When compared to the 14-moment approximation in ref. [1] in the presence of a magnetic field, the bulk viscous relaxation equation did not have any magnetic field dependent term.

For diffusion current. The expression for the diffusion current for the net charge in second order is:

$$
V_{(2)}^{\mu}=\Delta_{\alpha}^{\mu} \int d p p^{\alpha}\left(\delta \tilde{f}^{(2)}-\delta \tilde{\bar{f}}^{(2)}\right),
$$

where $\delta \tilde{f}_{(2)}$ is taken from eq. (3.2). Like other dissipative quantities, the total diffusion four vector is composed of first and second order terms, i.e., $V^{\mu}=V_{(1)}^{\mu}+V_{(2)}^{\mu}$. After evaluating the integral (for details see appendix B.3 in eq. (3.24), we get the following second order evolution equation for the diffusion current:

$$
\begin{aligned}
& \frac{V^{\mu}}{\tau_{c}}=-\dot{V}^{\langle\mu\rangle}-V_{\nu} \omega^{\nu \mu}-\lambda_{V V} V^{\nu} \sigma_{\nu}^{\mu}-\delta_{V V} V^{\mu} \theta+\lambda_{V \Pi} \Pi \nabla^{\mu} \alpha-\lambda_{V \pi} \pi^{\mu \nu} \nabla_{\nu} \alpha-\tau_{V \pi} \pi_{\nu}^{\mu} u^{\nu} \\
& +\tau_{V \Pi \Pi} \Pi \dot{u}^{\mu}+l_{V \pi} \Delta^{\mu \nu} \partial_{\gamma} \pi_{\nu}^{\gamma}-l_{V \Pi} \nabla^{\mu} \Pi+\beta_{V} \nabla^{\mu} \alpha-q B \delta_{V B} b^{\mu \gamma} V_{\gamma}+\tau_{c} q B l_{V \pi B} b^{\sigma \mu} \partial^{\kappa} \pi_{\kappa \sigma} \\
& +\tau_{c} q B \tau_{V \Pi B} b^{\gamma \mu} \Pi \dot{u}_{\gamma}-\tau_{c} q B l_{V \Pi B} b^{\gamma \mu} \nabla_{\gamma} \Pi-q \tau_{c} \delta_{V V B} B b^{\mu \nu} V_{\nu} \theta-q \tau_{c} \lambda_{V V B} B b^{\gamma \nu} V_{\nu} \sigma_{\gamma}^{\mu} \\
& -q \tau_{c} \rho_{V V B} B b^{\gamma \nu} V_{\nu} \omega_{\gamma}^{\mu}-\tau_{c} q \tau_{V V B} \Delta_{\gamma}^{\mu} D\left(B b^{\gamma \nu} V_{\nu}\right) \text {, }
\end{aligned}
$$




\begin{tabular}{|l|l|l|}
\hline $\begin{array}{l}\text { Transport } \\
\text { Coefficients }\end{array}$ & $\begin{array}{l}\text { Denicol } \\
\text { et al. }\end{array}$ & CE \\
\hline$\beta_{\pi}$ & $4 P / 5$ & $4 P / 5$ \\
\hline$\tau_{\pi \pi}$ & $10 / 7$ & $10 / 7$ \\
\hline$\delta_{\pi \pi}$ & $4 / 3$ & $4 / 3$ \\
\hline$\tau_{\pi V}$ & 0 & 0 \\
\hline$\lambda_{\pi V}$ & 0 & 0 \\
\hline$l_{\pi V}$ & 0 & 0 \\
\hline
\end{tabular}

(a)

\begin{tabular}{|l|l|l|}
\hline $\begin{array}{l}\text { Transport } \\
\text { Coefficients }\end{array}$ & $\begin{array}{l}\text { Denicol } \\
\text { et al. }\end{array}$ & CE \\
\hline$\beta_{V}$ & $n_{f} / 12$ & $n_{f} / 12$ \\
\hline$\lambda_{V V}$ & $3 / 5$ & $3 / 5$ \\
\hline$\delta_{V V}$ & 1 & 1 \\
\hline$\tau_{V \pi}$ & $\beta / 20$ & $\beta / 4$ \\
\hline$\lambda_{V \pi}$ & $\beta / 20$ & $\beta / 16$ \\
\hline$l_{V \pi}$ & $\beta / 20$ & $\beta / 4$ \\
\hline
\end{tabular}

(b)

\begin{tabular}{|l|l|l|}
\hline $\begin{array}{l}\text { Transport } \\
\text { Coefficients }\end{array}$ & $\begin{array}{l}\text { Denicol } \\
\text { et al. }\end{array}$ & CE \\
\hline$\delta_{\pi B}$ & $2 \beta / 5$ & $\beta / 2$ \\
\hline$\delta_{V B}$ & $5 \beta / 12$ & $\beta$ \\
\hline$\delta_{\pi V B}$ & - & $2 / 5$ \\
\hline$\delta_{\Pi V B}$ & - & $1 / 3$ \\
\hline$\tau_{\Pi V B}$ & - & $2 / 3$ \\
\hline$l_{V \pi B}$ & - & $\beta^{2} / 12$ \\
\hline$\tau_{V \Pi B}$ & - & $\beta^{2} / 12$ \\
\hline$l_{V \Pi B}$ & - & $\beta^{2} / 12$ \\
\hline$\delta_{V V B}$ & - & $\beta / 3$ \\
\hline$\lambda_{V V B}$ & - & $3 \beta / 20$ \\
\hline$\rho_{V V B}$ & - & $\beta / 4$ \\
\hline$\tau_{V V B}$ & - & $\beta / 4$ \\
\hline
\end{tabular}

(c)

Table 1. (a) Comparison between the coefficients for the shear-stress equation for a massless Boltzmann gas (here we compare the result for particles only) calculated in this work using CE method and Denicol et al. using the 14-moment method in ref. [65]. (b) Comparison between the coefficients for the diffusion equation for a massless Boltzmann gas calculated in this work using Chapman-Enskog method (CE) and Denicol et al. using the 14-moment method in ref. [65] (particles only). (c) Transport coefficients appearing in the shear, bulk and diffusion equation that couple magnetic field and dissipative quantities for a massless Boltzmann gas (particles only).

where the second order transport coefficients are given in terms of thermodynamic integrals in table 4. Coefficients $\tau_{V \pi}, \lambda_{V \pi}$ contain the derivative of $l_{V \pi} ; \tau_{V \Pi}, \lambda_{V \Pi}$ contain the derivative of $l_{V \Pi}$ and $\delta_{V V B}$ contains derivative of $\tau_{V V B}$, respectively. To arrive at the final expression eq. (3.25) we also make use of $\mathcal{X}$ and $\mathcal{Y}$ given in eq. (3.17). A comparison of the above to the RTA calculation done in ref. [63] without magnetic field shows that the last eight terms in the r.h.s. are new and are magnetic field dependent. A similar comparison with the relaxation equation for the diffusion in the presence of a magnetic field derived in ref. [1] using 14-moment approximation shows that the first 13 terms are of similar form, while the last seven terms are not present in the moment method. 


\subsection{The ultrarelativistic and weak field limit}

The transport coefficients in the ultra-relativistic limit, i.e. $m / T=0$, for a classical Maxwell gas with a constant relaxation time $\tau_{c}$, can be calculated analytically using the thermodynamic integrals. The transport coefficients are grouped into: (i) those which are independent of the magnetic field are collected in tables 1(a) and 1(b) for the shear and the number diffusion, respectively. (ii) Those which are magnetic field dependent are collected in table 1(c). In this limit, the bulk viscous pressure vanishes and has not been considered. In the same table, the results from the 14-moment approximation in the presence of a magnetic field ref. [1] in the ultra-relativistic limit have also been shown. It is worthwhile to note that in this limit, the new coefficients namely $\delta_{\pi B}$ and $\delta_{V B}$ are different in the above two approaches.

In the limit of weak magnetic field, which translates to the statement that temperature of the system is sufficiently large than the strength of the magnetic field $T^{2} \gg q B$. We define the dimensionless parameter $g_{B}=q B / T^{2}$ such that $g_{B} \ll 1$. The RTA approximation in the presence of magnetic field eq. (3.5), has two power counting schemes, viz. $\mathrm{Kn}=\tau_{c} T$ and $\chi=q B \tau_{c} / T$. However, in the weak field limit, the expansion parameter $\chi=g_{B} \tau_{c} T$ becomes smaller and hence treated as sub-leading contribution. Therefore, at second order one effectively retains term till $\mathcal{O}\left(\mathrm{Kn}^{2}\right)$ in spatial gradients and $\mathcal{O}(\chi \cdot \mathrm{Kn})$ for the mixed terms. ${ }^{1}$ In this limit the relaxation equations reduce to following forms:

$$
\begin{aligned}
& \dot{\pi}^{\mu \nu}=2 \beta_{\pi} \sigma^{\mu \nu}-\frac{\pi^{\mu \nu}}{\tau_{c}}+2 \pi_{\gamma}^{\langle\mu} \omega^{\nu\rangle \gamma}-\tau_{\pi \pi} \pi_{\gamma}^{\langle\mu} \sigma^{\nu\rangle \gamma}-\delta_{\pi \pi} \pi^{\mu \nu} \theta+\lambda_{\pi \Pi} \Pi \sigma^{\mu \nu}-\tau_{\pi V} V^{\langle\mu} \dot{u}^{\nu\rangle} \\
& +\lambda_{\pi V} V^{\langle\mu} \nabla^{\nu\rangle} \alpha+l_{\pi V} \nabla^{\langle\mu} V^{\nu\rangle}+\delta_{\pi B} \Delta_{\eta \beta}^{\mu \nu} q B b^{\gamma \eta} g^{\beta \rho} \pi_{\gamma \rho}, \\
& \dot{\Pi}=-\beta_{\Pi} \theta-\frac{\Pi}{\tau_{c}}-\delta_{\Pi \Pi} \Pi \theta+\lambda_{\Pi \pi} \pi^{\mu \nu} \sigma_{\mu \nu}-\tau_{\Pi V} V \cdot \dot{u}-\lambda_{\Pi V} V \cdot \nabla \alpha-l_{\Pi V} \partial \cdot V, \\
& \dot{V}^{\langle\mu\rangle}=\beta_{V} \nabla^{\mu} \alpha-\frac{V^{\mu}}{\tau_{c}}-V_{\nu} \omega^{\nu \mu}-\lambda_{V V} V^{\nu} \sigma_{\nu}^{\mu}-\delta_{V V} V^{\mu} \theta+\lambda_{V \Pi} \Pi \nabla^{\mu} \alpha-\lambda_{V \pi} \pi^{\mu \nu} \nabla_{\nu} \alpha \\
& -\tau_{V \pi} \pi_{\nu}^{\mu} \dot{u}^{\nu}+\tau_{V \Pi} \Pi u^{\mu}+l_{V \pi} \Delta^{\mu \nu} \partial_{\gamma} \pi_{\nu}^{\gamma}-l_{V \Pi} \nabla^{\mu} \Pi-q B \delta_{V B} b^{\mu \gamma} V_{\gamma} .
\end{aligned}
$$

The above set of reduced relaxation equations (3.26)-(3.28) formally corresponds to the relaxation equations as given in ref. [1]. The dimensionless magnetic field dependent transport coefficients $T \delta_{\pi B}$ and $T \delta_{V B}$ in the weak field limit are plotted in figure 1 as a function of $m / T$. In the limit $m / T \rightarrow 0$, these coefficients reduce to those obtained in table 1 (c).

\subsection{The Navier-Stokes limit}

In the Navier-Stokes limit, we keep terms $\mathcal{O}(\mathrm{Kn})$, and $\mathcal{O}(\chi \cdot \mathrm{Kn})$, which leaves us with the first and second terms in right hand side of eqs. (3.26)-(3.28) which are of first-order in gradients as well as the last term which is magnetic field dependent and are also first-order

\footnotetext{
${ }^{1}$ We do not keep terms which are $\mathcal{O}\left(\chi^{2}\right)$, since they do not contribute to the expansion eq. (3.5).
} 


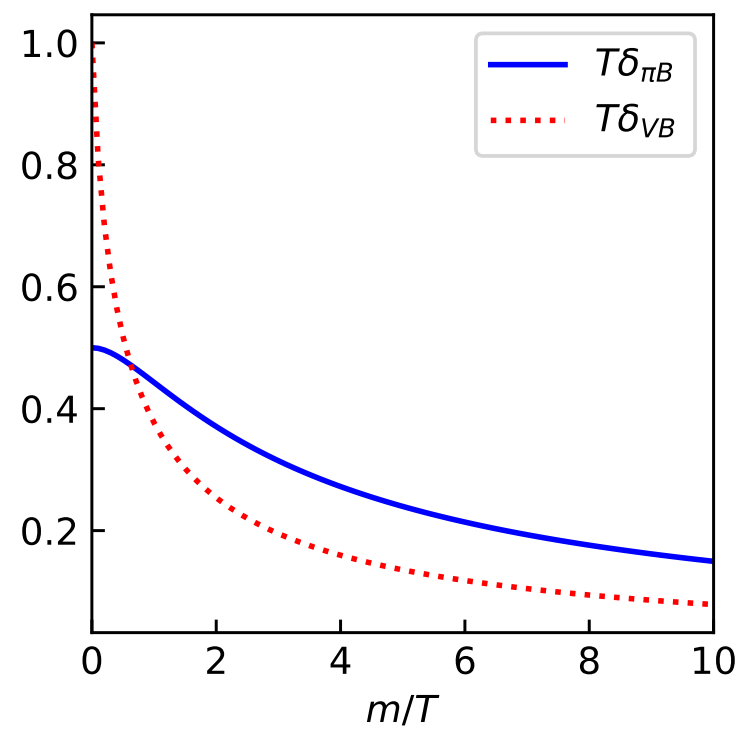

Figure 1. Dimensionless transport coefficients $T \delta_{V B}$ and $T \delta_{\pi B}$ that couple fluid to magnetic field as a function of $m / T$.

in gradients. Bringing these terms to the left we have,

$$
\begin{aligned}
\frac{\Pi}{\tau_{c}} & =\beta_{\Pi} \theta, \\
\left(\frac{g^{\mu \nu}}{\tau_{c}}+q B \delta_{V B} b^{\mu \gamma}\right) V_{\gamma} & =\beta_{V} \nabla^{\mu} \alpha, \\
\left(\frac{g^{\mu \gamma} g^{\nu \rho}}{\tau_{c}}-\delta_{\pi B} \Delta_{\eta \beta}^{\mu \nu} q B b^{\gamma \eta} g^{\beta \rho}\right) \pi_{\gamma \rho} & =2 \beta_{\pi} \sigma^{\mu \nu} .
\end{aligned}
$$

Since the bulk viscous pressure does not involve any magnetic field dependence, the Navier-Stokes limit for bulk viscosity $\zeta$ turns out to be trivially the same as that without any magnetic field, i.e., $\zeta=\beta_{\Pi} \tau_{c}$. One needs now to invert the coefficients multiplied in the left of the rest of the equations to get the respective constitutive relations. The general solution for the rest of the equations are given as

$$
\begin{aligned}
V_{\gamma}= & \left(\kappa_{\|} P_{\delta \gamma}^{\|}+\kappa_{\perp} P_{\delta \gamma}^{\perp}+\kappa_{\times} P_{\delta \gamma}^{\times}\right) \partial^{\delta} \alpha \\
\pi_{\gamma \rho}= & \left(\eta_{0} P_{\alpha \beta \gamma \rho}^{(0)}+\eta_{1}\left(P_{\alpha \beta \gamma \rho}^{(1)}+P_{\alpha \beta \gamma \rho}^{(-1)}\right)+i \eta_{2}\left(P_{\alpha \beta \gamma \rho}^{(1)}-P_{\alpha \beta \gamma \rho}^{(-1)}\right)+\eta_{3}\left(P_{\alpha \beta \gamma \rho}^{(2)}+P_{\alpha \beta \gamma \rho}^{(-2)}\right)\right. \\
& \left.\quad+i \eta_{4}\left(P_{\alpha \beta \gamma \rho}^{(2)}-P_{\alpha \beta \gamma \rho}^{(-2)}\right)\right) \sigma^{\alpha \beta} .
\end{aligned}
$$

where $P_{\delta \gamma}^{\|}, P_{\delta \gamma}^{\perp}$ and $P_{\delta \gamma}^{\times}$are second rank projection tensors while $P_{\alpha \beta \gamma \rho}^{(n)}$, with $n=-2$ to $n=+2$ are fourth rank projection tensors, respectively, where as the symbol $i=\sqrt{-1}$. The definition of these tensors are shown in appendix $\mathrm{C}$ and further details can be found in refs. [80, 81]. In the above set of equations $\kappa_{\|}, \kappa_{\perp}, \kappa_{\times}$and $\eta_{0}-\eta_{4}$ are the transport coefficients. These coefficients can be obtained by substituting the above solution to the 

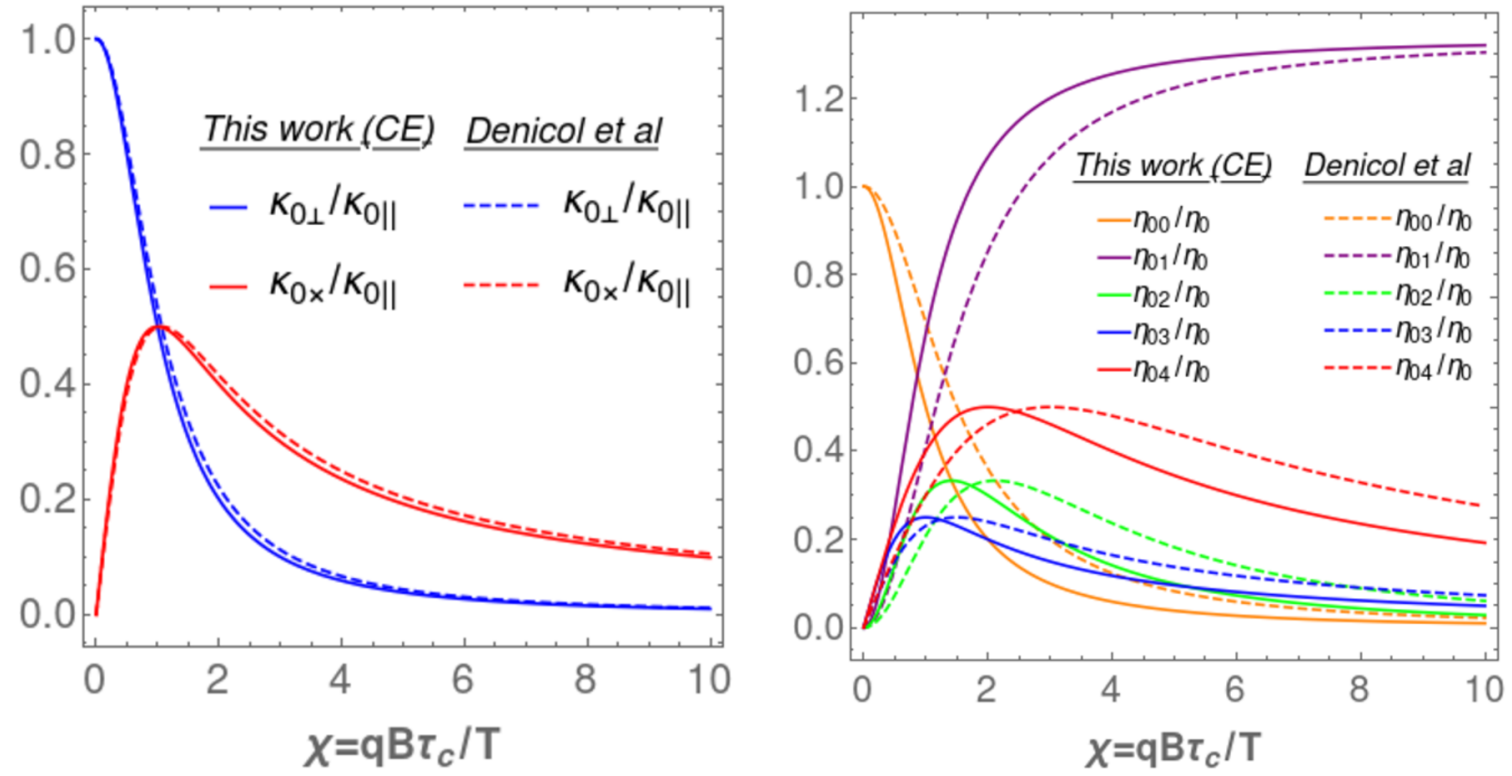

Figure 2. The magnetic-field dependence of the diffusion coefficients and the shear-viscosity coefficients. The solid lines are the results from the current work while the dotted lines are from ref. [1].

left hand side of eq. (3.31) and eq. (3.30) and using the usual properties of projection tensors. The resulting diffusion coefficients are

$$
\begin{aligned}
\kappa_{\|} & =\beta_{V} \tau_{c}, \\
\kappa_{\perp} & =\frac{\beta_{V} \tau_{c}}{1+\left(q B \tau_{c} \delta_{V B}\right)^{2}}, \\
\kappa_{\times} & =\frac{\beta_{V} q B \tau_{c}^{2} \delta_{V B}}{1+\left(q B \tau_{c} \delta_{V B}\right)^{2}}=\kappa_{\perp} q B \tau_{c} \delta_{V B} .
\end{aligned}
$$

Similarly, the shear viscous coefficients are

$$
\begin{aligned}
\eta_{0} & =2 \beta_{\pi} \tau_{c} \\
\eta_{1} & =\frac{2 \beta_{\pi} \tau_{c}}{1+\left(2 q B \tau_{c} \delta_{\pi B}\right)^{2}}, \\
\eta_{2} & =\frac{4 \beta_{\pi} q B \tau_{c}^{2} \delta_{\pi B}}{1+\left(2 q B \tau_{c} \delta_{\pi B}\right)^{2}}=2 \eta_{1} q B \tau_{c} \delta_{\pi B}, \\
\eta_{3} & =\frac{2 \beta_{\pi} \tau_{c}}{1+\left(q B \tau_{c} \delta_{\pi B}\right)^{2}}, \\
\eta_{4} & =\frac{2 \beta_{\pi} q B \tau_{c}^{2} \delta_{\pi B}}{1+\left(q B \tau_{c} \delta_{\pi B}\right)^{2}}=\eta_{3} q B \tau_{c} \delta_{\pi B} .
\end{aligned}
$$

The coefficients $\eta_{1}$ and $\eta_{3}$ are even function of magnetic field, where as $\eta_{2}$ and $\eta_{4}$ may have either sign, and they are odd functions of $B$. In the limit of vanishing magnetic field, i.e., $q B \rightarrow 0$, the diffusion coefficients reduce to $\kappa_{\times} \rightarrow 0$ and $\kappa_{\|}=\kappa_{\perp}$, while the shear viscous coefficients reduce to $\eta_{2}=\eta_{4}=0$ and $\eta_{1}=\eta_{3}=\eta_{0}$ as expected. 
The transport coefficients in the Navier-Stokes limit found in the present work are $\kappa_{\|}$, $\kappa_{\perp}$ and $\kappa_{\times}$for the diffusion coefficients and $\eta_{0}, \eta_{1}, \eta_{2}, \eta_{3}, \eta_{4}$ for the shear viscous coefficients respectively. In ref. [1] the above were labelled as $\kappa_{0 \|}, \kappa_{0 \perp}$ and $\kappa_{0 \times}$ for the diffusion coefficients and $\eta_{00}, \eta_{01}, \eta_{02}, \eta_{03}, \eta_{04}$ for the shear viscous coefficients respectively. Given one uses the same basis as ref. [1], we get the following relations between the transport coefficients [82]:

$$
\begin{aligned}
\kappa_{0 \|} & \equiv \kappa_{\|}, \\
\kappa_{0 \perp} & \equiv \kappa_{\perp}, \\
\kappa_{0 \times} & \equiv \kappa_{\times}, \\
\eta_{00} & \equiv \eta_{1} \\
\eta_{01} & \equiv \frac{16\left(q B \tau_{c} \delta_{\pi B}\right)^{2}}{3} \eta_{1}, \\
\eta_{02} & \equiv \eta_{3}-\eta_{1}, \\
\eta_{03} & \equiv \eta_{2} / 2, \\
\eta_{04} & \equiv \eta_{4} .
\end{aligned}
$$

The transport coefficients from both the approaches, in the $m=0$ limit, is shown in figure 2 as a function of $\chi=q B \tau_{c} / T$. The solid lines correspond to the results from RTA used in the present work while the dotted lines are the results from 14-moment approximation used in ref. [1]. One notices that although quantitatively the transport coefficients are different moreover qualitatively they are similar. The difference arises because one obtains different value of transport coefficients $\delta_{\pi B}$ and $\delta_{V B}$ in the two approaches.

\section{Conclusion}

We derive for the first time the relativistic non-resistive, viscous second-order magnetohydrodynamics equations for the dissipative quantities using the relaxation time approximation. Assuming that the single-particle distribution function is close to equilibrium, we solve the Boltzmann equation in the presence of a magnetic field using Chapman-Enskog like gradient expansion with two relevant expansion parameters: the Knudsen number and a dimensionless parameter $\chi=q B \tau_{c} / T$ that depends on the strength of the magnetic field. In first-order, dissipative quantities are found to be independent of the magnetic field. Moreover, in second-order, we found new transport coefficients that couple magnetic field to dissipative quantities apart from the usual transport coefficients that one gets without any external field. When compared to the results of the 14-moment approximation, additional terms involving the magnetic field appear in the relaxation time approximation. However, in the weak field limit, the form of the relaxation equations is the same as that of the 14-moment approximation but with different values for the transport coefficients. In the ultra-relativistic limit, the resulting transport coefficients from the two approaches are compared, some of the coefficients are found to differ. Finally, we find that one recovers the usual anisotropic transport coefficients for fluid in magnetic fields in the Navier-Stokes limit. As a further extension of the present work, we plan to investigate the general case for 
the relativistic resistive viscous fluid in a magnetic field in a future study in ref. [83]. The formulation of the relativistic causal magnetohydrodynamics is expected to be useful in astrophysical phenomena involving relativistic plasmas as well as femto-scopic high energy heavy-ion collisions.

\section{Acknowledgments}

AP acknowledges the CSIR-HRDG financial support. AD and VR acknowledges support from the DAE, Govt. of India. RB and VR acknowledge financial support from the DST Inspire faculty research grant (IFA-16-PH-167), India.

\section{A Thermodynamic integrals}

The $n$-th moments integral for the distribution function is defined as:

$$
I_{\mu_{1} \mu_{2} \cdots \mu_{n}}^{(m) \pm}=\int \frac{d p}{(u \cdot p)^{m}} p_{\mu_{1}} p_{\mu_{2}} \cdots p_{\mu_{n}}\left(f_{0} \pm \bar{f}_{0}\right)
$$

which can be docomposed as:

$$
\begin{aligned}
I_{\mu_{1} \mu_{2} \ldots \mu_{n}}^{(m) \pm}= & I_{n 0}^{(m) \pm} u_{\mu_{1}} \cdots u_{\mu_{n}}+I_{n 1}^{(m) \pm}\left(\Delta_{\mu_{1} \mu_{2}} u_{\mu_{3}} \cdots u_{\mu_{n}}+\text { perm. }\right)+\cdots \\
& \cdots+I_{n q}^{(m) \pm}\left(\Delta_{\mu_{1} \mu_{2}} \Delta_{\mu_{3} \mu_{4}} \cdots \Delta_{\mu_{n-1} \mu_{n}}+\text { perm. }\right) .
\end{aligned}
$$

where $n \geq 2 q$.

Similarly the auxiliary moments integral

$$
J_{\mu_{1} \mu_{2} \cdots \mu_{n}}^{(m) \pm}=\int \frac{d p}{(u \cdot p)^{m}} p_{\mu_{1}} p_{\mu_{2}} \cdots p_{\mu_{n}}\left(f_{0} \tilde{f}_{0} \pm \bar{f}_{0} \tilde{\bar{f}}_{0}\right)
$$

can be decomposed as:

$$
\begin{aligned}
J_{\mu_{1} \mu_{2} \ldots \mu_{n}}^{(m) \pm}= & J_{n 0}^{(m) \pm} u_{\mu_{1}} \cdots u_{\mu_{n}}+J_{n 1}^{(m) \pm}\left(\Delta_{\mu_{1} \mu_{2}} u_{\mu_{3}} \cdots u_{\mu_{n}}+\text { perm. }\right)+\cdots \\
& \cdots+J_{n q}^{(m) \pm}\left(\Delta_{\mu_{1} \mu_{2}} \Delta_{\mu_{3} \mu_{4}} \cdots \Delta_{\mu_{n-1} \mu_{n}}+\text { perm. }\right)
\end{aligned}
$$

where $\tilde{f}_{0}=1-r f_{0}$. Here we define the thermodynamic integrals as follows:

$$
I_{n q}^{(m) \pm}=\frac{1}{(2 q+1) ! !} \int d p(u \cdot p)^{n-2 q-m}\left(\Delta_{\alpha \beta} p^{\alpha} p^{\beta}\right)^{q}\left(f_{0} \pm \bar{f}_{0}\right),
$$

and

$$
J_{n q}^{(m) \pm}=\frac{1}{(2 q+1) ! !} \int d p(u \cdot p)^{n-2 q-m}\left(\Delta_{\alpha \beta} p^{\alpha} p^{\beta}\right)^{q}\left(f_{0} \tilde{f}_{0} \pm \bar{f}_{0} \tilde{\tilde{f}}_{0}\right) .
$$

One can write the $J$ in terms of $I$ as:

$$
J_{n q}^{(0) \pm}=\frac{1}{\beta}\left[-I_{n-1, q-1}^{(0) \pm}+(n-2 q) I_{n-1, q}^{(0) \pm}\right] .
$$

The general expression of $D_{n q}$ used in eq. (2.41) and eq. (2.42) is given by: $D_{n q}=$ $J_{n+1, q}^{(0)+} J_{n-1, q}^{(0)+}-J_{n q}^{(0)-} J_{n q}^{(0)-}$. 


\section{B Second order relaxation equation for dissipative stresses}

In this appendix we discuss the detail calculation of the second order dissipative stresses. The contribution due to the antiparticles are not shown explicitly for simplicity but they appear in the final expressions.

\section{B.1 Shear stress}

The second order shear stress $\pi_{(2)}^{\mu \nu}$ is given by eq. (3.19):

$$
\pi_{(2)}^{\mu \nu}=\Delta_{\alpha \beta}^{\mu \nu} \int d p p^{\alpha} p^{\beta}\left(\frac{\tau_{c}}{u \cdot p} p^{\rho} \partial_{\rho}\left[\frac{\tau_{c}}{u \cdot p} p^{\sigma} \partial_{\sigma} f_{0}\right]+\frac{\tau_{c}}{u \cdot p} q F^{\gamma \eta} p_{\eta} \frac{\partial}{\partial p^{\gamma}}\left[\frac{\tau_{c}}{u \cdot p} p^{\sigma} \partial_{\sigma} f_{0}\right]\right) .
$$

For convenience, we write them into two parts as:

$$
\pi_{(2)}^{\mu \nu}=\mathcal{I}_{1}+\mathcal{I}_{2}
$$

Here

$$
\begin{aligned}
& \mathcal{I}_{1}=\Delta_{\alpha \beta}^{\mu \nu} \int d p p^{\alpha} p^{\beta}\left(\frac{\tau_{c}}{u \cdot p} p^{\rho} \partial_{\rho}\left[\frac{\tau_{c}}{u \cdot p} p^{\sigma} \partial_{\sigma} f_{0}\right]\right), \\
& \mathcal{I}_{2}=\Delta_{\alpha \beta}^{\mu \nu} \int d p p^{\alpha} p^{\beta}\left(\frac{\tau_{c}}{u \cdot p} q F^{\gamma \eta} p_{\eta} \frac{\partial}{\partial p^{\gamma}}\left[\frac{\tau_{c}}{u \cdot p} p^{\sigma} \partial_{\sigma} f_{0}\right]\right),
\end{aligned}
$$

Let us first evaluate the integral $\mathcal{I}_{1}$ :

$$
\begin{aligned}
\mathcal{I}_{1} & =\Delta_{\alpha \beta}^{\mu \nu} \int d p p^{\alpha} p^{\beta}\left(\frac{\tau_{c}}{u \cdot p} p^{\rho} \partial_{\rho}\left[\frac{\tau_{c}}{u \cdot p} p^{\sigma} \partial_{\sigma} f_{0}\right]\right), \\
& =\mathcal{A}+\mathcal{B}+\mathcal{C}
\end{aligned}
$$

where

$$
\begin{aligned}
\mathcal{A} & =\Delta_{\alpha \beta}^{\mu \nu} \int d p p^{\alpha} p^{\beta} \tau_{c} D\left[\frac{\tau_{c}}{u \cdot p} p^{\sigma} \partial_{\sigma} f_{0}\right], \\
\mathcal{B} & =\Delta_{\alpha \beta}^{\mu \nu} \int d p p^{\alpha} p^{\beta} \frac{\tau_{c}}{u \cdot p} p^{\rho} \nabla_{\rho}\left[\tau_{c} \dot{f}_{0}\right] \\
\mathcal{C} & =\Delta_{\alpha \beta}^{\mu \nu} \int d p p^{\alpha} p^{\beta} \frac{\tau_{c}}{u \cdot p} p^{\rho} \nabla_{\rho}\left[\frac{\tau_{c}}{u \cdot p} p^{\sigma} \nabla_{\sigma} f_{0}\right] .
\end{aligned}
$$

A straight forward calculation gives:

$$
\mathcal{A}=-\Delta_{\alpha \beta}^{\mu \nu} \int d p f_{0} \tilde{f}_{0} p^{\alpha} p^{\beta} \tau_{c} D\left[\frac{\tau_{c}}{u \cdot p} p^{\sigma}\left\{\beta p^{\gamma} \partial_{\sigma} u_{\gamma}+(u \cdot p) \partial_{\sigma} \beta-\partial_{\sigma} \alpha\right\}\right] .
$$

We can rewrite the above expression in terms of the thermodynamic integrals given in appendix A and eq. (2.43) as:

$$
\begin{aligned}
\mathcal{A}= & -\tau_{c} \dot{\pi}^{\langle\mu \nu\rangle}-2 \tau_{c}^{2}\left(\frac{n_{f}}{\epsilon+P} J_{31}^{(0)-}-J_{31}^{(1)-}\right) \dot{u}^{\langle\mu} \nabla^{\nu\rangle} \alpha+\tau_{c}^{2} \Delta_{\alpha \beta}^{\mu \nu} J_{31}^{(0)+} \dot{u}^{\beta}\left[\frac{\beta q B b^{\alpha \sigma}}{\epsilon+P} V_{\sigma}\right] \\
& +\tau_{c}^{2} \Delta_{\alpha \beta}^{\mu \nu} J_{31}^{(0)+} \dot{u}^{\alpha}\left[\frac{\beta q B b^{\beta \sigma}}{\epsilon+P} V_{\sigma}\right] .
\end{aligned}
$$


Similarly for $\mathcal{B}$ we have:

$$
\begin{aligned}
\mathcal{B} & =\Delta_{\alpha \beta}^{\mu \nu} \int d p p^{\alpha} p^{\beta} \frac{\tau_{c}}{u \cdot p} p^{\rho} \nabla_{\rho}\left[\tau_{c} \dot{f}_{0}\right] \\
& =-\Delta_{\alpha \beta}^{\mu \nu} \int d p f_{0} \tilde{f}_{0} p^{\alpha} p^{\beta} \frac{\tau_{c}}{u \cdot p} p^{\rho} \nabla_{\rho} \tau_{c}\left[\beta p^{\gamma} \dot{u}_{\gamma}+(u \cdot p) \dot{\beta}-\dot{\alpha}\right] .
\end{aligned}
$$

Using the thermodynamics integral discussed in appendix A we get:

$$
\begin{aligned}
\mathcal{B} & =-2 \tau_{c}^{2}\left[\left(J_{31}^{(0)+}+J_{42}^{(1)+}\right) \dot{\beta}-\left(J_{31}^{(1)-}+J_{42}^{(2)-}\right) \dot{\alpha}\right] \sigma^{\mu \nu}-2 \tau_{c}^{2} \nabla^{\langle\mu}\left(\dot{u}^{\nu\rangle} \beta J_{42}^{(1)+}\right), \\
& =-2 \tau_{c}^{2}\left[\left(J_{31}^{(0)+}+J_{42}^{(1)+}\right) \mathcal{X}-\left(J_{31}^{(1)-}+J_{42}^{(2)-}\right) \mathcal{Y}\right] \theta \sigma^{\mu \nu}-2 \tau_{c}^{2} \nabla^{\langle\mu}\left(\dot{u}^{\nu\rangle} \beta J_{42}^{(1)+}\right),
\end{aligned}
$$

where in the last line we have used the expression for $\dot{\alpha}$ and $\dot{\beta}$ given in eq. (2.41) and eq. (2.42). The $\mathcal{X}$ and $\mathcal{Y}$ are same as eq. (3.17). Finally, for $\mathcal{C}$ we have

$$
\begin{aligned}
\mathcal{C} & =\Delta_{\alpha \beta}^{\mu \nu} \int d p p^{\alpha} p^{\beta} \frac{\tau_{c}}{u \cdot p} p^{\rho} \nabla_{\rho}\left[\frac{\tau_{c}}{u \cdot p} p^{\sigma} \nabla_{\sigma} f_{0}\right], \\
& =-\Delta_{\alpha \beta}^{\mu \nu} \int d p f_{0} \tilde{f}_{0} p^{\alpha} p^{\beta} \frac{\tau_{c}}{u \cdot p} p^{\rho} \nabla_{\rho}\left[\frac{\tau_{c}}{u \cdot p} p^{\sigma}\left(\beta p^{\gamma} \nabla_{\sigma} u_{\gamma}+(u \cdot p) \nabla_{\sigma} \beta-\nabla_{\sigma} \alpha\right)\right] .
\end{aligned}
$$

Like the previous cases we use the thermodynamic integrals given in appendix A along with eq. (2.43) and eq. (1.1) to rewrite the above expression:

$$
\begin{aligned}
\mathcal{C}= & 2 \nabla^{\langle\mu}\left(\dot{u}^{\nu\rangle} \beta \tau_{c}^{2} J_{42}^{(1)+}\right)+2 \nabla^{\langle\mu}\left[\nabla^{\nu\rangle} \alpha \tau_{c}^{2}\left(J_{42}^{(2)-}-\frac{1}{h} J_{42}^{(1)-}\right)\right]-4 \beta \tau_{c}^{2}\left(2 J_{63}^{(3)+}+J_{42}^{(1)+}\right) \sigma_{\rho}^{\langle\mu} \sigma^{\nu\rangle \rho} \\
& -\frac{20}{3} \beta \tau_{c}^{2} J_{42}^{(1)+} \theta \sigma^{\mu \nu}-\frac{28}{3} \beta \tau_{c}^{2} J_{63}^{(3)+} \theta \sigma^{\mu \nu}-4 \beta \tau_{c}^{2}\left(J_{42}^{(1)+}+2 J_{63}^{(3)+}\right) \sigma^{\langle\mu \rho} \omega_{\rho}^{\nu\rangle} \\
& +2 \tau_{c}^{2} \nabla^{\langle\mu}\left[J_{42}^{(1)+}\left(\frac{\beta q B b^{\nu\rangle \gamma} V_{\gamma}}{\epsilon+P}\right)\right]
\end{aligned}
$$

Now let us evaluate the second integral $\mathcal{I}_{2}$ :

$$
\begin{aligned}
\mathcal{I}_{2} & =-\Delta_{\alpha \beta}^{\mu \nu} \int d p p^{\alpha} p^{\beta}\left(\left(\frac{\tau_{c}}{u \cdot p}\right)^{2} q B b^{\gamma \eta} p_{\eta} \frac{\partial}{\partial p^{\gamma}}\left[p^{\sigma} \partial_{\sigma} f_{0}\right]\right), \\
& =\Delta_{\alpha \beta}^{\mu \nu} \int d p f_{0} \tilde{f}_{0} p^{\alpha} p^{\beta}\left(\left(\frac{\tau_{c}}{u \cdot p}\right)^{2} q B b^{\gamma \eta} p_{\eta}\left(\left(\beta p^{\rho} \partial_{\sigma} u_{\rho}+(u \cdot p) \partial_{\sigma} \beta-\partial_{\sigma} \alpha\right) \Delta_{\gamma}^{\sigma}\right)+\beta \partial_{\sigma} u_{\rho} p^{\sigma} \Delta_{\gamma}^{\rho}\right), \\
& =2 \tau_{c}^{2} q B b^{\gamma \eta} \beta J_{42}^{(2)-}\left(\Delta_{\eta \beta}^{\mu \nu} g^{\beta \rho}+\Delta_{\alpha \eta}^{\mu \nu} g^{\alpha \rho}\right) \sigma_{\gamma \rho},
\end{aligned}
$$


where we have used $\frac{\partial}{\partial p^{\gamma}} p^{\sigma} \partial_{\sigma} f_{0}=\partial_{\sigma} f_{0} \Delta_{\gamma}^{\sigma}+p^{\sigma} \frac{\partial}{\partial p^{\gamma}} \partial_{\sigma} f_{0}$ and the expression for $\partial_{\sigma} f_{0}$ to arrive at the final expression. Now using eqs. (B.6)-(B.9) we get the final expression:

$$
\begin{aligned}
\pi_{(2)}^{\mu \nu}= & -\tau_{c} \dot{\pi}^{\langle\mu \nu\rangle}-2 \tau_{c}^{2} \dot{u}^{\langle\mu} \nabla^{\nu\rangle} \alpha\left(\frac{n_{f}}{\epsilon+P} J_{31}^{(0)-}-J_{31}^{(1)-}\right)+\tau_{c}^{2} \Delta_{\alpha \beta}^{\mu \nu} J_{31}^{(0)+} \dot{u}^{\beta}\left[\frac{\beta q B b^{\alpha \sigma}}{\epsilon+P} V_{\sigma}\right] \\
& +\tau_{c}^{2} \Delta_{\alpha \beta}^{\mu \nu} J_{31}^{(0)+} \dot{u}^{\alpha}\left[\frac{\beta q B b^{\beta \sigma}}{\epsilon+P} V_{\sigma}\right]-2 \tau_{c}^{2}\left[\left(J_{31}^{(0)+}+J_{42}^{(1)+}\right) \mathcal{X}-\left(J_{31}^{(1)-}+J_{42}^{(2)-}\right) \mathcal{Y}\right] \theta \sigma^{\mu \nu} \\
& -2 \tau_{c}^{2} \nabla^{\langle\mu}\left(\dot{u}^{\nu\rangle} \beta J_{42}^{(1)+}\right)+2 \nabla^{\langle\mu}\left(\dot{u}^{\nu\rangle} \beta \tau_{c}^{2} J_{42}^{(1)+}\right)+2 \nabla^{\langle\mu}\left[\nabla^{\nu\rangle} \alpha \tau_{c}^{2}\left(J_{42}^{(2)-}-\frac{n_{f}}{\epsilon+P} J_{42}^{(1)-}\right)\right] \\
& -\frac{20}{3} \beta \tau_{c}^{2} J_{42}^{(1)+} \theta \sigma^{\mu \nu}-4 \beta \tau_{c}^{2}\left(2 J_{63}^{(3)+}+J_{42}^{(1)+}\right) \sigma_{\rho}^{\langle\mu} \sigma^{\nu\rangle \rho}-\frac{28}{3} \beta \tau_{c}^{2} J_{63}^{(3)+} \theta \sigma^{\mu \nu} \\
& -4 \beta \tau_{c}^{2}\left(J_{42}^{(1)+}+2 J_{63}^{(3)+}\right) \sigma^{\langle\mu \rho} \omega_{\rho}^{\nu\rangle}+2 \tau_{c}^{2} \nabla^{\langle\alpha}\left[J_{42}^{(1)+}\left(\frac{\beta q B b^{\beta\rangle \gamma} V_{\gamma}}{\epsilon+P}\right)\right] \\
& +2 \tau_{c}^{2} q B b^{\gamma \eta} \beta J_{42}^{(2)-}\left(\Delta_{\eta \beta}^{\mu \nu} g^{\beta \rho}+\Delta_{\alpha \eta}^{\mu \nu} g^{\alpha \rho}\right) \sigma_{\gamma \rho} .
\end{aligned}
$$

Here we kept terms only upto second-order in gradients.

\section{B.2 Bulk stress}

Let us now consider the bulk viscous case. From eq. (3.22) we get:

$$
\begin{aligned}
\Pi_{(2)} & =-\frac{1}{3} \Delta_{\alpha \beta} \int d p p^{\alpha} p^{\beta}\left(\frac{\tau_{c}}{u \cdot p} p^{\mu} \partial_{\mu}\left[\frac{\tau_{c}}{u \cdot p} p^{\rho} \partial_{\rho} f_{0}\right]+\frac{\tau_{c}}{u \cdot p} q F^{\mu \nu} p_{\nu} \frac{\partial}{\partial p^{\mu}}\left[\frac{\tau_{c}}{u \cdot p} p^{\rho} \partial_{\rho} f_{0}\right]\right), \\
& =\mathcal{I}_{1}+\mathcal{I}_{2},
\end{aligned}
$$

where

$$
\begin{aligned}
& \mathcal{I}_{1}=-\frac{\Delta_{\alpha \beta}}{3} \int d p p^{\alpha} p^{\beta} \frac{\tau_{c}}{u \cdot p} p^{\mu} \partial_{\mu}\left[\frac{\tau_{c}}{u \cdot p} p^{\rho} \partial_{\rho} f_{0}\right] \\
& \mathcal{I}_{2}=-\frac{\Delta_{\alpha \beta}}{3} \int d p p^{\alpha} p^{\beta} \frac{\tau_{c}}{u \cdot p} q F^{\mu \nu} p_{\nu} \frac{\partial}{\partial p^{\mu}}\left[\frac{\tau_{c}}{u \cdot p} p^{\rho} \partial_{\rho} f_{0}\right] .
\end{aligned}
$$

Note that for our case $F^{\mu \nu}=-B b^{\mu \nu}$. Let us first evaluate $\mathcal{I}_{1}$ by breaking it into three parts $\mathcal{I}_{1}=\mathcal{A}+\mathcal{B}+\mathcal{C}$ where

$$
\begin{aligned}
\mathcal{A} & =-\frac{\Delta_{\alpha \beta}}{3} \int d p p^{\alpha} p^{\beta} \tau_{c} D\left[\frac{\tau_{c}}{u \cdot p} p^{\rho} \partial_{\rho} f_{0}\right], \\
\mathcal{B} & =-\frac{\Delta_{\alpha \beta}}{3} \int d p p^{\alpha} p^{\beta} \frac{\tau_{c}}{u \cdot p} p^{\mu} \nabla_{\mu}\left(\tau_{c} \dot{f}_{0}\right), \\
\mathcal{C} & =-\frac{\Delta_{\alpha \beta}}{3} \int d p p^{\alpha} p^{\beta} \frac{\tau_{c}}{u \cdot p} p^{\mu} \nabla_{\mu}\left(\frac{\tau_{c} p^{\rho}}{u \cdot p} \nabla_{\rho} f_{0}\right) .
\end{aligned}
$$

We evaluate each of the above integrals one-by-one:

$$
\begin{aligned}
\mathcal{A} & =-\frac{\Delta_{\alpha \beta}}{3} \int d p p^{\alpha} p^{\beta} \tau_{c} D\left[\frac{\tau_{c}}{u \cdot p} p^{\rho} \partial_{\rho} f_{0}\right] \\
& =\frac{\Delta_{\alpha \beta}}{3} \int d p f_{0} \tilde{f}_{0} p^{\alpha} p^{\beta} \tau_{c} D\left[\frac{\tau_{c}}{u \cdot p} p^{\rho}\left(\beta p^{\gamma} \partial_{\rho} u_{\gamma}+(u \cdot p) \partial_{\rho} \beta-\partial_{\rho} \alpha\right)\right] \\
& =-\tau_{c} \dot{\Pi}+\frac{2 \tau_{c}^{2}}{3} J_{31}^{(0)-} \frac{n_{f}}{\epsilon+P} \nabla^{\alpha} \alpha \dot{u}_{\alpha}-\frac{2 \tau_{c}^{2}}{3} J_{21}^{(0)-} \nabla^{\alpha} \alpha \dot{u}_{\alpha}-\frac{2 \tau_{c}^{2} \beta}{3(\epsilon+P)} J_{31}^{(0)+} \dot{u}_{\alpha} q B b^{\alpha \beta} V_{\beta}
\end{aligned}
$$


We have used the thermodynamic integrals given in appendix A along with eq. (A.2) and eq. (2.43) to arrive at the final expression eq. (B.12). Now let us evaluate $\mathcal{B}$ with the help of thermodynamic integrals and its properties given in appendix A:

$$
\begin{aligned}
\mathcal{B} & =-\frac{\Delta_{\alpha \beta}}{3} \int d p p^{\alpha} p^{\beta} \frac{\tau_{c}}{u \cdot p} p^{\mu} \nabla_{\mu}\left(\tau_{c} \dot{f}_{0}\right) \\
& =\frac{5 \tau_{c}^{2}}{3} \nabla_{\mu}\left(\beta J_{42}^{(1)+} \dot{u}^{\mu}\right)+\frac{5 \tau_{c}^{2}}{3} \theta\left[\left(J_{31}^{(0)+}+J_{42}^{(1)+}\right) \dot{\beta}-\left(J_{31}^{(1)-}+J_{42}^{(2)-}\right) \dot{\alpha}\right] .
\end{aligned}
$$

Similarly for $\mathcal{C}$ we have

$$
\begin{aligned}
\mathcal{C}= & -\frac{\Delta_{\alpha \beta}}{3} \int d p p^{\alpha} p^{\beta} \frac{\tau_{c}}{u \cdot p} p^{\mu} \nabla_{\mu}\left(\frac{\tau_{c} p^{\rho}}{u \cdot p} \nabla_{\rho} f_{0}\right), \\
= & \frac{\Delta_{\alpha \beta}}{3} \int d p f_{0} \tilde{f}_{0} p^{\alpha} p^{\beta} \frac{\tau_{c}}{u \cdot p} p^{\mu} \nabla_{\mu}\left(\frac{\tau_{c} p^{\rho}}{u \cdot p}\left(\beta p^{\gamma} \nabla_{\rho} u_{\gamma}+(u \cdot p) \nabla_{\rho} \beta-\nabla_{\rho} \alpha\right)\right), \\
= & \frac{5 \tau_{c}^{2} \beta}{9}\left(7 J_{63}^{(3)+}+\frac{23}{3} J_{42}^{(1)+}\right) \theta^{2}+\frac{5 \tau_{c}^{2}}{3} \nabla_{\mu}\left[\nabla^{\mu} \alpha\left(J_{42}^{(1)-} \frac{n_{f}}{\epsilon+P}-J_{42}^{(2)-}\right)\right] \\
& +\frac{\tau_{c}^{2} \beta}{3}\left(7 J_{63}^{(3)+}+J_{42}^{(1)+}\right) \sigma^{\mu \nu} \sigma_{\mu \nu}+\frac{5 \tau_{c}^{2}}{3} \nabla_{\mu}\left[-J_{42}^{(1)+} \beta \dot{u}^{\mu}-\frac{J_{42}^{(1)+} \beta q B b^{\mu \nu} V_{\nu}}{\epsilon+P}\right] .
\end{aligned}
$$

Needless to say, here we kept only terms upto the second-order. The remaining integral $\mathcal{I}_{2}$ is evaluated in a similar fashion,

$$
\begin{aligned}
\mathcal{I}_{2} & =\frac{\Delta_{\alpha \beta}}{3} \int d p p^{\alpha} p^{\beta} \frac{\tau_{c}}{u \cdot p} q B b^{\mu \nu} p_{\nu} \frac{\partial}{\partial p^{\mu}}\left[\frac{\tau_{c}}{u \cdot p} p^{\rho} \partial_{\rho} f_{0}\right] \\
& =-\frac{\Delta_{\alpha \beta}}{3} \int d p f_{0} \tilde{f}_{0} p^{\alpha} p^{\beta} \frac{\tau_{c}}{u \cdot p} q B b^{\mu \nu} p_{\nu} \frac{\partial}{\partial p^{\mu}}\left[\frac{\tau_{c}}{u \cdot p} p^{\rho}\left(p^{\gamma} \beta \partial_{\rho} u_{\gamma}+(u \cdot p) \partial_{\rho} \beta-\partial_{\rho} \alpha\right)\right] \\
& =-\frac{\Delta_{\alpha \beta}}{3} \tau_{c}^{2} q B b_{\nu}^{\mu}\left(\beta J_{(2)-}^{\alpha \beta \nu \gamma} \nabla_{\mu} u_{\gamma}+\beta J_{(2)-}^{\alpha \beta \nu \rho} \partial_{\rho} u_{\mu}\right)
\end{aligned}
$$

using the expansion given in eq. (A.2) and the anti-symmetric property of $b^{\mu \nu}$ we get:

$$
\mathcal{I}_{2}=-\frac{q B \tau_{c}^{2}}{3} \beta J_{42}^{(2)-}\left(5 b^{\mu \gamma} \nabla_{\mu} u_{\gamma}+5 b^{\mu \rho} \nabla_{\rho} u_{\mu}\right)=0 .
$$

Finally using eqs. (B.12)-(B.14) and eq. (B.16) we have:

$$
\begin{aligned}
\Pi_{(2)}= & -\tau_{c} \dot{\Pi}+\frac{2 \tau_{c}^{2}}{3 h} J_{31}^{(0)-} \dot{u}_{\alpha} \nabla^{\alpha} \alpha-\frac{2 \tau_{c}^{2}}{3} J_{21}^{(0)-} \dot{u}_{\alpha} \nabla^{\alpha} \alpha-\frac{2 \tau_{c}^{2} \beta}{3(\epsilon+P)} J_{31}^{(0)+} \dot{u}_{\alpha} q B b^{\alpha \beta} V_{\beta} \\
& +\frac{5 \tau_{c}^{2} \beta}{9}\left(7 J_{63}^{(3)+}+\frac{23}{3} J_{42}^{(1)+}\right) \theta^{2}+\frac{5 \tau_{c}^{2}}{3}\left[\left(J_{31}^{(0)+}+J_{42}^{(1)+}\right) \dot{\beta}-\left(J_{31}^{(1)-}+J_{42}^{(2)-}\right) \dot{\alpha}\right] \theta \\
& +\frac{\tau_{c}^{2} \beta}{3}\left(7 J_{63}^{(3)+}+J_{42}^{(1)+}\right) \sigma^{\mu \nu} \sigma_{\mu \nu}+\frac{5 \tau_{c}^{2}}{3} \nabla_{\mu}\left[\left(\frac{1}{h} J_{42}^{(1)-}-J_{42}^{(2)-}\right) \nabla^{\mu} \alpha\right] \\
& -\frac{5 \tau_{c}^{2}}{3} \nabla_{\mu}\left[\frac{J_{42}^{(1)+} \beta q B b^{\mu \nu} V_{\nu}}{\epsilon+P}\right] .
\end{aligned}
$$

Eq. (B.17) is the second-order relaxation equation for the bulk-viscous stress. 


\section{B.3 Diffusion current}

In this section we discuss the detail derivation of the diffusion current. From eq. (3.24) we get:

$$
V_{(2)}^{\mu}=\Delta_{\alpha}^{\mu} \int d p p^{\alpha}\left(\frac{\tau_{c}}{u \cdot p} p^{\sigma} \partial_{\sigma}\left[\frac{\tau_{c}}{u \cdot p} p^{\rho} \partial_{\rho} f_{0}\right]+\frac{\tau_{c}}{u \cdot p} q F^{\sigma \nu} p_{\nu} \frac{\partial}{\partial p^{\sigma}}\left[\frac{\tau_{c}}{u \cdot p} p^{\rho} \partial_{\rho} f_{0}\right]\right),
$$

where

$$
\begin{aligned}
& \mathcal{I}_{1}=\Delta_{\alpha}^{\mu} \int d p p^{\alpha} \frac{\tau_{c}}{u \cdot p} p^{\sigma} \partial_{\sigma}\left[\frac{\tau_{c}}{u \cdot p} p^{\rho} \partial_{\rho} f_{0}\right] \\
& \mathcal{I}_{2}=\Delta_{\alpha}^{\mu} \int d p p^{\alpha} \frac{\tau_{c}}{u \cdot p} q F^{\sigma \nu} p_{\nu} \frac{\partial}{\partial p^{\sigma}}\left[\frac{\tau_{c}}{u \cdot p} p^{\rho} \partial_{\rho} f_{0}\right] .
\end{aligned}
$$

Let us first calculate the $\mathcal{I}_{1}$ by breaking it up into three parts as: $\mathcal{I}_{1}=\mathcal{A}+\mathcal{B}+\mathcal{C}$ where

$$
\begin{aligned}
\mathcal{A} & =\Delta_{\alpha}^{\mu} \int d p p^{\alpha} \tau_{c} D\left[\frac{\tau_{c}}{u \cdot p} p^{\rho} \partial_{\rho} f_{0}\right], \\
\mathcal{B} & =\Delta_{\alpha}^{\mu} \int d p p^{\alpha} \frac{\tau_{c}}{u \cdot p} p^{\sigma} \nabla_{\sigma}\left(\tau_{c} \dot{f}_{0}\right), \\
\mathcal{C} & =\Delta_{\alpha}^{\mu} \int d p p^{\alpha} \frac{\tau_{c}}{u \cdot p} p^{\sigma} \nabla_{\sigma}\left(\frac{\tau_{c} p^{\rho}}{u \cdot p} \nabla_{\rho} f_{0}\right) .
\end{aligned}
$$

For $\mathcal{A}$ we get:

$$
\begin{aligned}
\mathcal{A} & =-\Delta_{\alpha}^{\mu} \int d p f_{0} \tilde{f}_{0} p^{\alpha} \tau_{c} D\left[\frac{\tau_{c}}{u \cdot p} p^{\rho}\left(\beta p^{\gamma} \partial_{\rho} u_{\gamma}+(u \cdot p) \partial_{\rho} \beta-\partial_{\rho} \alpha\right)\right], \\
& =-\Delta_{\alpha}^{\mu} D\left[\int d p f_{0} \tilde{f}_{0} p^{\alpha} \tau_{c} \frac{\tau_{c}}{u \cdot p} p^{\rho}\left(\beta p^{\gamma} \partial_{\rho} u_{\gamma}+(u \cdot p) \partial_{\rho} \beta-\partial_{\rho} \alpha\right)\right], \\
& =-\tau_{c} \dot{V}^{\langle\mu\rangle}-\tau_{c}^{2} \Delta_{\gamma}^{\mu} D\left[\frac{n_{f} q B b^{\gamma \nu} V_{\nu}}{\epsilon+P}\right] .
\end{aligned}
$$

We have used the thermodynamic integrals and its expansion given in the appendix A, along with eqs. (2.41) and (2.42) to arrive at the final expression. Similarly for $\mathcal{B}$ we get:

$$
\begin{aligned}
\mathcal{B}= & \Delta_{\alpha}^{\mu} \int d p p^{\alpha} \frac{\tau_{c}}{u \cdot p} p^{\sigma} \nabla_{\sigma}\left(\tau_{c} \dot{f}_{0}\right) \\
= & \Delta_{\alpha}^{\mu} \nabla_{\sigma}\left(\int d p p^{\alpha} \frac{\tau_{c}}{u \cdot p} p^{\sigma} \tau_{c} \dot{f}_{0}\right)+\Delta_{\alpha}^{\mu} \nabla_{\sigma} u_{\gamma}\left(\int d p p^{\alpha} p^{\gamma} \frac{\tau_{c}}{(u \cdot p)^{2}} p^{\sigma} \tau_{c} \dot{f}_{0}\right) \\
= & -\tau_{c}^{2} \nabla^{\mu}\left(J_{21}^{(0)-} \dot{\beta}-J_{21}^{(1)+} \dot{\alpha}\right)-\tau_{c}^{2} \beta \dot{u}^{\mu} \theta\left(\frac{4}{3} J_{21}^{(0)-}+\frac{5}{3} J_{42}^{(2)-}\right)-\tau_{c}^{2} \beta J_{21}^{(0)-} \dot{u}_{\gamma} \omega^{\gamma \mu} \\
& -\tau_{c}^{2} \beta \dot{u}_{\gamma} \sigma^{\gamma \mu}\left(J_{21}^{(0)-}+2 J_{42}^{(2)-}\right) .
\end{aligned}
$$

Lastly for $\mathcal{C}$ we get:

$$
\begin{aligned}
\mathcal{C} & =\Delta_{\alpha}^{\mu} \int d p p^{\alpha} \frac{\tau_{c}^{2}}{u \cdot p} p^{\sigma} \nabla_{\sigma}\left(\frac{p^{\rho}}{u \cdot p} \nabla_{\rho} f_{0}\right) \\
& =\Delta_{\alpha}^{\mu} \nabla_{\sigma}\left(\int d p p^{\alpha} \frac{\tau_{c}^{2}}{u \cdot p} p^{\sigma} \frac{p^{\rho}}{u \cdot p} \nabla_{\rho} f_{0}\right)+\Delta_{\alpha}^{\mu} \nabla_{\sigma} u_{\gamma}\left(\int d p p^{\alpha} p^{\gamma} \frac{\tau_{c}^{2}}{(u \cdot p)^{2}} p^{\sigma} \frac{p^{\rho}}{u \cdot p} \nabla_{\rho} f_{0}\right) .
\end{aligned}
$$


Substituting the expression for $\nabla_{\rho} f_{0}$ and using the usual thermodynamic integrals and their expansion along with eq. (2.43) and eq. (1.1) the above expression takes the following form:

$$
\begin{aligned}
\mathcal{C}= & -\frac{4 \tau_{c}^{2}}{3}\left(\frac{J_{21}^{(0)+} n_{f}}{\epsilon+P}-J_{21}^{(1)+}\right)\left(\nabla^{\mu} \alpha\right) \theta+\frac{4 \tau_{c}^{2}}{3} J_{21}^{(0)-} \beta \dot{u}^{\mu} \theta-\tau_{c}^{2}\left(\frac{J_{21}^{(0)+} n_{f}}{\epsilon+P}-J_{21}^{(1)+}\right)\left(\nabla^{\gamma} \alpha\right) \sigma_{\gamma}^{\mu} \\
& +\tau_{c}^{2} J_{21}^{(0)-} \beta \dot{u}^{\gamma} \sigma_{\gamma}^{\mu}-\tau_{c}^{2}\left(\frac{J_{21}^{(0)+} n_{f}}{\epsilon+P}-J_{21}^{(1)+}\right)\left(\nabla^{\gamma} \alpha\right) \omega_{\gamma}^{\mu}+\tau_{c}^{2} J_{21}^{(0)-} \beta \dot{u}^{\gamma} \omega_{\gamma}^{\mu} \\
& +\tau_{c} J_{21}^{(0)-} \omega_{\gamma}^{\mu}\left[\frac{\beta q B b^{\gamma \nu} V_{\nu}}{\epsilon+P}\right]-2 \tau_{c}^{2}\left(\frac{J_{42}^{(2)+} n_{f}}{\epsilon+P}-J_{42}^{(3)+}\right)\left(\nabla^{\gamma} \alpha\right) \sigma_{\gamma}^{\mu}+2 \tau_{c}^{2} J_{42}^{(2)-} \beta \dot{u}^{\gamma} \sigma_{\gamma}^{\mu} \\
& -\frac{5 \tau_{c}^{2}}{3}\left(\frac{J_{42}^{(2)+} n_{f}}{\epsilon+P}-J_{42}^{(3)+}\right)\left(\nabla^{\mu} \alpha\right) \theta+\frac{5 \tau_{c}^{2}}{3} J_{42}^{(2)-} \beta \dot{u}^{\mu} \theta-2 \tau_{c}^{2} \Delta_{\rho}^{\mu} \nabla_{\gamma}\left(\beta J_{42}^{(2)-} \sigma^{\rho \gamma}\right) \\
& -\frac{5 \tau_{c}^{2}}{3} \nabla^{\mu}\left[\beta J_{42}^{(2)-} \theta\right]+\frac{4 \tau_{c}}{3} J_{21}^{(0)-} \theta\left[\frac{\beta q B b^{\mu \nu} V_{\nu}}{\epsilon+P}\right]+\tau_{c} J_{21}^{(0)-} \sigma_{\gamma}^{\mu}\left[\frac{\beta q B b^{\gamma \nu} V_{\nu}}{\epsilon+P}\right] \\
& +2 \tau_{c} J_{42}^{(2)-} \sigma_{\gamma}^{\mu}\left[\frac{\beta q B b^{\gamma \nu} V_{\nu}}{\epsilon+P}\right]+\frac{5 \tau_{c}}{3} J_{42}^{(2)-} \theta\left[\frac{\beta q B b^{\mu \nu} V_{\nu}}{\epsilon+P}\right] .
\end{aligned}
$$

Now let us calculate the integral $\mathcal{I}_{2}$ :

$$
\begin{gathered}
\mathcal{I}_{2}=-\Delta_{\alpha}^{\mu} \int d p f_{0} \tilde{f}_{0} p^{\alpha} \frac{\tau_{c}}{u \cdot p} q F^{\sigma \nu} p_{\nu} \frac{\partial}{\partial p^{\sigma}}\left[\frac{\tau_{c}}{u \cdot p} p^{\rho}\left(\beta p^{\gamma} \partial_{\rho} u_{\gamma}+(u \cdot p) \partial_{\rho} \beta-\partial_{\rho} \alpha\right)\right], \\
=\tau_{c}^{2} q B\left[\frac{1}{h} J_{21}^{(1)-} b^{\gamma \mu} \nabla_{\gamma} \alpha-J_{21}^{(2)-} b^{\gamma \mu} \nabla_{\gamma} \alpha\right. \\
\left.\quad-\frac{\beta J_{21}^{(1)+} b^{\gamma \mu} \Delta_{\gamma}^{\sigma} \partial^{k} \pi_{k \sigma}}{\epsilon+P}-\frac{\beta J_{21}^{(1)+} b^{\gamma \mu} \Pi \dot{u}_{\gamma}}{\epsilon+P}+\frac{\left.\beta J_{21}^{(1)+} b^{\gamma \mu} \nabla_{\gamma} \Pi\right]}{\epsilon+P}\right] .
\end{gathered}
$$

Adding eqs. (B.19)-(B.22) together we get the final expression for the diffusion current (which after simplification becomes eq. (3.25)):

$$
\begin{aligned}
V_{(2)}^{\mu}= & -\tau_{c} \dot{V}^{\langle\mu\rangle}-\tau_{c}^{2} \Delta_{\gamma}^{\mu} D\left[\frac{n_{f} q B b^{\gamma \nu} V_{\nu}}{\epsilon+P}\right]-\tau_{c}^{2} \nabla^{\mu}\left(J_{21}^{(0)-} \dot{\beta}-J_{21}^{(1)+} \dot{\alpha}\right) \\
& -\tau_{c}^{2} \beta \dot{u}^{\mu} \theta\left(\frac{4}{3} J_{21}^{(0)-}+\frac{5}{3} J_{42}^{(2)-}\right)-\tau_{c}^{2} \beta J_{21}^{(0)-} \dot{u}_{\gamma} \omega^{\gamma \mu}-\tau_{c}^{2} \beta \dot{u}_{\gamma} \sigma^{\gamma \mu}\left(J_{21}^{(0)-}+2 J_{42}^{(2)-}\right) \\
& -\frac{4 \tau_{c}^{2}}{3}\left(\frac{J_{21}^{(0)+} n_{f}}{\epsilon+P}-J_{21}^{(1)+}\right)\left(\nabla^{\mu} \alpha\right) \theta+\frac{4 \tau_{c}^{2}}{3} J_{21}^{(0)-} \beta \dot{u}^{\mu} \theta-\tau_{c}^{2}\left(\frac{J_{21}^{(0)+} n_{f}}{\epsilon+P}-J_{21}^{(1)+}\right)\left(\nabla^{\gamma} \alpha\right) \sigma_{\gamma}^{\mu} \\
& +\tau_{c}^{2} J_{21}^{(0)-} \beta \dot{u}^{\gamma} \sigma_{\gamma}^{\mu}+2 \tau_{c}^{2} J_{42}^{(2)-} \beta \dot{u}^{\gamma} \sigma_{\gamma}^{\mu}-\tau_{c}^{2}\left(\frac{J_{21}^{(0)+} n_{f}}{\epsilon+P}-J_{21}^{(1)+}\right)\left(\nabla^{\gamma} \alpha\right) \omega_{\gamma}^{\mu}+\tau_{c}^{2} J_{21}^{(0)-} \beta \dot{u}^{\gamma} \omega_{\gamma}^{\mu} \\
& -2 \tau_{c}^{2}\left(\frac{J_{42}^{(2)+} n_{f}}{\epsilon+P}-J_{42}^{(3)+}\right)\left(\nabla^{\gamma} \alpha\right) \sigma_{\gamma}^{\mu}-\frac{5 \tau_{c}^{2}}{3}\left(\frac{J_{42}^{(2)+} n_{f}}{\epsilon+P}-J_{42}^{(3)+}\right)\left(\nabla^{\mu} \alpha\right) \theta \\
& +\frac{5 \tau_{c}^{2}}{3} J_{42}^{(2)-} \beta \dot{u}^{\mu} \theta-2 \tau_{c}^{2} \Delta_{\rho}^{\mu} \nabla_{\gamma}\left(\beta J_{42}^{(2)-} \sigma^{\rho \gamma}\right)-\frac{5 \tau_{c}^{2}}{3} \nabla^{\mu}\left[\beta J_{42}^{(2)-} \theta\right]+\frac{4 \tau_{c}}{3} J_{21}^{(0)-} \theta\left[\frac{\beta q B b^{\mu \nu} V_{\nu}}{\epsilon+P}\right] \\
& +\tau_{c} J_{21}^{(0)-} \sigma_{\gamma}^{\mu}\left[\frac{\beta q B b^{\gamma \nu} V_{\nu}}{\epsilon+P}\right]+2 \tau_{c} J_{42}^{(2)-} \sigma_{\gamma}^{\mu}\left[\frac{\beta q B b^{\gamma \nu} V_{\nu}}{\epsilon+P}\right]+\frac{5 \tau_{c}}{3} J_{42}^{(2)-} \theta\left[\frac{\beta q B b^{\mu \nu} V_{\nu}}{\epsilon+P}\right]
\end{aligned}
$$




$$
\begin{aligned}
+\tau_{c} J_{21}^{(0)-} \omega_{\gamma}^{\mu}\left[\frac{\beta q B b^{\gamma \nu} V_{\nu}}{\epsilon+P}\right]+\tau_{c}^{2} q B\left[\frac{J_{21}^{(1)-} b^{\gamma \mu}}{h} \nabla_{\gamma} \alpha-J_{21}^{(2)-} b^{\gamma \mu} \nabla_{\gamma} \alpha\right. \\
\left.-\frac{\beta J_{21}^{(1)+} b^{\gamma \mu} \Delta_{\gamma}^{\sigma} \partial^{k} \pi_{k \sigma}}{\epsilon+P}-\frac{\beta J_{21}^{(1)+} b^{\gamma \mu} \Pi \dot{u}_{\gamma}}{\epsilon+P}+\frac{\beta J_{21}^{(1)+} b^{\gamma \mu} \nabla_{\gamma} \Pi}{\epsilon+P}\right] .
\end{aligned}
$$

\section{Projection tensors}

The definition of the second and fourth rank projection tensors used in the text is shown in this section.

A general antisymmetric second rank tensor $b_{\mu \nu}$ can be defined by

$$
b_{\mu \nu} \equiv \varepsilon_{\mu \lambda \nu} b^{\lambda},
$$

where $b_{\mu}$ is a unit axial four vector. The second rank projection tensors are then defined as

$$
\begin{aligned}
P_{\mu \nu}^{(0)} & =b_{\mu} b_{\nu}, \\
P_{\mu \nu}^{(+1)} & =\frac{1}{2}\left(\Delta_{\mu \nu}-b_{\mu} b_{\nu}-i b_{\mu \nu}\right), \\
P_{\mu \nu}^{(-1)} & =\frac{1}{2}\left(\Delta_{\mu \nu}-b_{\mu} b_{\nu}+i b_{\mu \nu}\right) .
\end{aligned}
$$

where $i=\sqrt{-1}$. They satisfy the following properties

$$
\begin{aligned}
P_{\mu \kappa}^{(\mathrm{m})} P_{\nu}^{\left(\mathrm{m}^{\prime}\right), \kappa} & =\delta_{\mathrm{mm}^{\prime}} P_{\mu \nu}^{(\mathrm{m})}, \\
\left(P_{\mu \nu}^{(\mathrm{m})}\right)^{\dagger} & =P_{\mu \nu}^{(-\mathrm{m})}=P_{\nu \mu}^{(\mathrm{m})}, \\
\sum_{\mathrm{m}=-1}^{1} P_{\mu \nu}^{(\mathrm{m})} & =\Delta_{\mu \nu}, \quad P_{\mu \mu}^{(\mathrm{m})}=1,
\end{aligned}
$$

where $\mathrm{m}, \mathrm{m}^{\prime}=0, \pm 1$. The projection tensors $P_{\mu \nu}^{(\mathrm{m})}$ satisfy the following eigenvalue equation (see ref. [80])

$$
P_{\mu \kappa}^{(\mathrm{m})} b_{\nu}^{\kappa}=i \mathrm{~m} P_{\mu \nu}^{(\mathrm{m})},
$$

where $m$ is the eigenvalue. Also $b_{\mu \nu}$ can be represented as a linear combination of the projection tensors

$$
b_{\mu \nu}=\sum_{\mathrm{m}=-1}^{1} i \mathrm{~m} P_{\mu \nu}^{(\mathrm{m})}
$$

It is also easy to generalize them to the fourth rank projection tensor which are defined in terms of the second rank projection tensor as

$$
P_{\mu \nu, \mu^{\prime} \nu^{\prime}}^{(\mathrm{m})}=\sum_{\mathrm{m}_{1}=-1}^{1} \sum_{\mathrm{m}_{2}=-1}^{1} P_{\mu \mu^{\prime}}^{\left(\mathrm{m}_{1}\right)} P_{\nu \nu^{\prime}}^{\left(\mathrm{m}_{2}\right)} \delta\left(\mathrm{m}, \mathrm{m}_{1}+\mathrm{m}_{2}\right),
$$

where $\delta\left(\mathrm{m}, \mathrm{m}_{1}+\mathrm{m}_{2}\right)=1$ for $\mathrm{m}=\mathrm{m}_{1}+\mathrm{m}_{2}$ and zero otherwise. Notice that $\mathrm{m}_{1}+\mathrm{m}_{2}$ assumes the five values $\mathrm{m}=-2,-1,0,1,2$ which in turn result in the five shear viscous coefficients dicussed in the text. A generalization to higher ranks is also possible using the above basis if need arises. 


\section{General expressions of transport coefficients}

\begin{tabular}{|l|l|}
\hline$\tau_{\pi \pi}$ & $\frac{2 \beta}{\beta_{\pi}}\left(2 J_{63}^{(3)+}+J_{42}^{(1)+}\right)$ \\
\hline$\delta_{\pi \pi}$ & $\frac{\beta}{3 \beta_{\pi}}\left(7 J_{63}^{(3)+}+5 J_{42}^{(1)+}\right)$ \\
\hline$\lambda_{\pi \Pi}$ & $\frac{2}{\beta_{\Pi}}\left[\left(J_{31}^{(0)+}+J_{42}^{(1)+}\right) \mathcal{X}-\left(J_{31}^{(1)-}+J_{42}^{(2)-}\right) \mathcal{Y}+\frac{\beta}{3}\left(7 J_{63}^{(3)+}+5 J_{42}^{(1)+}\right)\right]$ \\
\hline$l_{\pi V}$ & $\frac{2}{\beta_{V}}\left(J_{42}^{(2)-}-\frac{n_{f}}{\epsilon+P} J_{42}^{(1)-}\right)$ \\
\hline$\delta_{\pi B}$ & $2 J_{42}^{(2)-} / J_{42}^{(1)+}$ \\
\hline$\delta_{\pi V B}$ & $2 \beta J_{42}^{(1)+} /(\epsilon+P)$ \\
\hline
\end{tabular}

Table 2. Transport coefficients appearing in shear-stress equation eq. (3.21).

\begin{tabular}{|l|l|}
\hline$\delta_{\Pi \Pi}$ & $\frac{5}{3 \beta_{\Pi}}\left[\left(J_{31}^{(0)+}+J_{42}^{(1)+}\right) \mathcal{X}-\left(J_{31}^{(1)-}+J_{42}^{(2)-}\right) \mathcal{Y}+\frac{\beta}{3}\left(7 J_{63}^{(3)+}+\right.\right.$ \\
$\left.\left.\frac{23}{5} J_{42}^{(1)+}\right)\right]$
\end{tabular}

Table 3. Transport coefficients appearing in bulk equation eq. (3.23).

\begin{tabular}{|l|l|}
\hline$\lambda_{V V}$ & $1+\frac{2}{\beta_{V}}\left(\frac{n_{f}}{\epsilon+P} J_{42}^{(2)+}-J_{42}^{(3)+}\right)$ \\
\hline$\delta_{V V}$ & $\frac{4}{3}+\frac{5}{3 \beta_{V}}\left(\frac{n_{f} J_{42}^{(2)+}}{\epsilon+P}-J_{42}^{(3)+}\right)$ \\
\hline$l_{V \pi}$ & $-\frac{\beta}{\beta_{\pi}} J_{42}^{(2)-}$ \\
\hline$\tau_{V \pi}$ & $-\beta \frac{\partial}{\partial \beta} l_{V \pi}$ \\
\hline$\lambda_{V \pi}$ & $-\frac{1}{h} \frac{\partial}{\partial \beta} l_{V \pi}$ \\
\hline$l_{V \Pi}$ & $-\frac{1}{\beta_{\Pi}}\left(\mathcal{X} J_{21}^{(0)-}-\mathcal{Y} J_{21}^{(1)+}+\frac{5 \beta}{3} J_{42}^{(1)-}\right)$ \\
\hline$\delta_{V B}$ & $-\left(\frac{n_{f} J_{21}^{(1)-}}{\epsilon+P}-J_{21}^{(2)-}\right) / \beta_{V}$ \\
\hline$l_{V \pi B}$ & $-\beta J_{21}^{(1)+} /(\epsilon+P)$ \\
\hline$\tau_{V \Pi B}$ & $-\beta J_{21}^{(1)+} /(\epsilon+P)$ \\
\hline$l_{V \Pi B}$ & $-\beta J_{21}^{(1)+} /(\epsilon+P)$ \\
\hline$\lambda_{V V B}$ & $-\frac{\beta}{\epsilon+P}\left(J_{21}^{(0)-}+2 J_{42}^{(2)-}\right)$ \\
\hline$\rho_{V V B}$ & $-\beta J_{21}^{(0)-} /(\epsilon+P)$ \\
\hline$\tau_{V V B}$ & $n_{f} /(\epsilon+P)$ \\
\hline
\end{tabular}

Table 4. Transport coefficients appearing in diffusion equation eq. (3.25). 
Open Access. This article is distributed under the terms of the Creative Commons Attribution License (CC-BY 4.0), which permits any use, distribution and reproduction in any medium, provided the original author(s) and source are credited.

\section{References}

[1] G.S. Denicol et al., Nonresistive dissipative magnetohydrodynamics from the Boltzmann equation in the 14-moment approximation, Phys. Rev. D 98 (2018) 076009 [arXiv: 1804.05210] [INSPIRE].

[2] A. Bzdak and V. Skokov, Event-by-event fluctuations of magnetic and electric fields in heavy ion collisions, Phys. Lett. B $\mathbf{7 1 0 ~ ( 2 0 1 2 )} 171$ [arXiv:1111.1949] [INSPIRE].

[3] W.-T. Deng and X.-G. Huang, Event-by-event generation of electromagnetic fields in heavy-ion collisions, Phys. Rev. C 85 (2012) 044907 [arXiv:1201.5108] [INSPIRE].

[4] K. Tuchin, Particle production in strong electromagnetic fields in relativistic heavy-ion collisions, Adv. High Energy Phys. 2013 (2013) 490495 [arXiv:1301.0099] [InSPIRE].

[5] V. Roy and S. Pu, Event-by-event distribution of magnetic field energy over initial fluid energy density in $\sqrt{s_{\mathrm{NN}}}=200 \mathrm{GeV}$ Au-Au collisions, Phys. Rev. C 92 (2015) 064902 [arXiv: 1508.03761] [INSPIRE].

[6] H. Li, X.-L. Sheng and Q. Wang, Electromagnetic fields with electric and chiral magnetic conductivities in heavy ion collisions, Phys. Rev. C 94 (2016) 044903 [arXiv:1602.02223] [INSPIRE].

[7] A.K. Chaudhuri, A short course on relativistic heavy ion collisions, IOPP, Bristol, U.K. (2014) [arXiv: 1207.7028] [INSPIRE].

[8] U. Heinz and R. Snellings, Collective flow and viscosity in relativistic heavy-ion collisions, Ann. Rev. Nucl. Part. Sci. 63 (2013) 123 [arXiv:1301.2826] [INSPIRE].

[9] C. Gale, S. Jeon and B. Schenke, Hydrodynamic modeling of heavy-ion collisions, Int. J. Mod. Phys. A 28 (2013) 1340011 [arXiv: 1301.5893] [INSPIRE].

[10] P. Romatschke and U. Romatschke, Relativistic fluid dynamics in and out of equilibrium, Cambridge University Press, Cambridge, U.K. (2019) [arXiv:1712.05815] [INSPIRE].

[11] S. Gupta, The electrical conductivity and soft photon emissivity of the QCD plasma, Phys. Lett. B 597 (2004) 57 [hep-lat/0301006] [INSPIRE].

[12] G. Aarts, C. Allton, A. Amato, P. Giudice, S. Hands and J.-I. Skullerud, Electrical conductivity and charge diffusion in thermal QCD from the lattice, JHEP 02 (2015) 186 [arXiv: 1412.6411] [INSPIRE].

[13] A. Amato, G. Aarts, C. Allton, P. Giudice, S. Hands and J.-I. Skullerud, Electrical conductivity of the quark-gluon plasma across the deconfinement transition, Phys. Rev. Lett. 111 (2013) 172001 [arXiv:1307.6763] [INSPIRE].

[14] X.-G. Huang, M. Huang, D.H. Rischke and A. Sedrakian, Anisotropic hydrodynamics, bulk viscosities and R-modes of strange quark stars with strong magnetic fields, Phys. Rev. D 81 (2010) 045015 [arXiv: 0910 .3633] [INSPIRE].

[15] X.-G. Huang, Electromagnetic fields and anomalous transports in heavy-ion collisions - a pedagogical review, Rept. Prog. Phys. 79 (2016) 076302 [arXiv:1509.04073] [InSPIRE]. 
[16] M. Greif, C. Greiner and Z. Xu, Magnetic field influence on the early time dynamics of heavy-ion collisions, Phys. Rev. C 96 (2017) 014903 [arXiv:1704.06505] [INSPIRE].

[17] V. Roy, S. Pu, L. Rezzolla and D.H. Rischke, Effect of intense magnetic fields on reduced-MHD evolution in $\sqrt{s_{\mathrm{NN}}}=200 \mathrm{GeV}$ Au+Au collisions, Phys. Rev. C 96 (2017) 054909 [arXiv: 1706.05326] [INSPIRE].

[18] U. Gürsoy, D. Kharzeev and K. Rajagopal, Magnetohydrodynamics, charged currents and directed flow in heavy ion collisions, Phys. Rev. C 89 (2014) 054905 [arXiv:1401.3805] [INSPIRE].

[19] G. Inghirami, L. Del Zanna, A. Beraudo, M.H. Moghaddam, F. Becattini and M. Bleicher, Numerical magneto-hydrodynamics for relativistic nuclear collisions, Eur. Phys. J. C 76 (2016) 659 [arXiv: 1609.03042] [INSPIRE].

[20] X.-G. Huang, A. Sedrakian and D.H. Rischke, Kubo formulae for relativistic fluids in strong magnetic fields, Annals Phys. 326 (2011) 3075 [arXiv:1108.0602] [INSPIRE].

[21] V. Roy, S. Pu, L. Rezzolla and D. Rischke, Analytic Bjorken flow in one-dimensional relativistic magnetohydrodynamics, Phys. Lett. B 750 (2015) 45 [arXiv:1506.06620] [INSPIRE].

[22] S. Pu, V. Roy, L. Rezzolla and D.H. Rischke, Bjorken flow in one-dimensional relativistic magnetohydrodynamics with magnetization, Phys. Rev. D 93 (2016) 074022 [arXiv: 1602.04953] [INSPIRE].

[23] I. Siddique, R.-J. Wang, S. Pu and Q. Wang, Anomalous magnetohydrodynamics with longitudinal boost invariance and chiral magnetic effect, Phys. Rev. D 99 (2019) 114029 [arXiv: 1904.01807] [INSPIRE].

[24] S. Pu and D.-L. Yang, Transverse flow induced by inhomogeneous magnetic fields in the Bjorken expansion, Phys. Rev. D 93 (2016) 054042 [arXiv: 1602.04954] [InSPIRE].

[25] M. Shokri and N. Sadooghi, Evolution of magnetic fields from the $3+1$ dimensional self-similar and Gubser flows in ideal relativistic magnetohydrodynamics, JHEP 11 (2018) 181 [arXiv: 1807.09487] [INSPIRE].

[26] M. Shokri and N. Sadooghi, Novel self-similar rotating solutions of nonideal transverse magnetohydrodynamics, Phys. Rev. D 96 (2017) 116008 [arXiv:1705.00536] [InSPIRE].

[27] M.H. Moghaddam, B. Azadegan, A.F. Kord and W.M. Alberico, Non-relativistic approximate numerical ideal-magneto-hydrodynamics of $(1+1 D)$ transverse flow in Bjorken scenario, Eur. Phys. J. C 78 (2018) 255 [arXiv:1705.08192] [InSPIRE].

[28] P.B. Arnold, G.D. Moore and L.G. Yaffe, Transport coefficients in high temperature gauge theories. 1. Leading log results, JHEP 11 (2000) 001 [hep-ph/0010177] [INSPIRE].

[29] P.B. Arnold, G.D. Moore and L.G. Yaffe, Transport coefficients in high temperature gauge theories. 2. Beyond leading log, JHEP 05 (2003) 051 [hep-ph/0302165] [INSPIRE].

[30] S. Li and H.-U. Yee, Shear viscosity of the quark-gluon plasma in a weak magnetic field in perturbative QCD: leading log, Phys. Rev. D 97 (2018) 056024 [arXiv:1707.00795] [INSPIRE].

[31] S. Ghosh, B. Chatterjee, P. Mohanty, A. Mukharjee and H. Mishra, Impact of magnetic field on shear viscosity of quark matter in Nambu-Jona-Lasinio model, Phys. Rev. D 100 (2019) 034024 [arXiv: 1804.00812] [INSPIRE]. 
[32] S. Cao et al., Toward the determination of heavy-quark transport coefficients in quark-gluon plasma, Phys. Rev. C 99 (2019) 054907 [arXiv:1809.07894] [InSPIRE].

[33] M. Kurian, S. Mitra, S. Ghosh and V. Chandra, Transport coefficients of hot magnetized QCD matter beyond the lowest Landau level approximation, Eur. Phys. J. C 79 (2019) 134 [arXiv: 1805.07313] [INSPIRE].

[34] B. Singh, L. Thakur and H. Mishra, Heavy quark complex potential in a strongly magnetized hot QGP medium, Phys. Rev. D 97 (2018) 096011 [arXiv:1711.03071] [INSPIRE].

[35] S. Bhadury, M. Kurian, V. Chandra and A. Jaiswal, Second order relativistic viscous hydrodynamics within an effective description of hot QCD medium, arXiv:2010.01537 [INSPIRE].

[36] S. Chakrabarty, Transport coefficients of quark-gluon plasma, Pramana 25 (1985) 673 [INSPIRE].

[37] C. Eckart, The thermodynamics of irreversible processes. 3. Relativistic theory of the simple fluid, Phys. Rev. 58 (1940) 919 [inSPIRE].

[38] L. Landau and E. Lifshitz, Fluid mechanics, volume 6, Elsevier Science, The Netherlands (2013).

[39] W.A. Hiscock and L. Lindblom, Stability and causality in dissipative relativistic fluids, Annals Phys. 151 (1983) 466 [inSPIRE].

[40] W.A. Hiscock and L. Lindblom, Generic instabilities in first-order dissipative relativistic fluid theories, Phys. Rev. D 31 (1985) 725 [InSPIRE].

[41] W.A. Hiscock and L. Lindblom, Linear plane waves in dissipative relativistic fluids, Phys. Rev. D 35 (1987) 3723 [INSPIRE].

[42] W. Israel, Nonstationary irreversible thermodynamics: a causal relativistic theory, Annals Phys. 100 (1976) 310 [INSPIRE].

[43] W. Israel and J.M. Stewart, Transient relativistic thermodynamics and kinetic theory, Annals Phys. 118 (1979) 341 [inSPIRE].

[44] S. Pu, T. Koide and D.H. Rischke, Does stability of relativistic dissipative fluid dynamics imply causality?, Phys. Rev. D 81 (2010) 114039 [arXiv:0907.3906] [INSPIRE].

[45] G.S. Denicol, T. Kodama, T. Koide and P. Mota, Shock propagation and stability in causal dissipative hydrodynamics, Phys. Rev. C 78 (2008) 034901 [arXiv:0805.1719] [InSPIRE].

[46] G.S. Denicol, T. Kodama, T. Koide and P. Mota, Stability and causality in relativistic dissipative hydrodynamics, J. Phys. G 35 (2008) 115102 [arXiv:0807.3120] [INSPIRE].

[47] S. Floerchinger and E. Grossi, Causality of fluid dynamics for high-energy nuclear collisions, JHEP 08 (2018) 186 [arXiv:1711.06687] [INSPIRE].

[48] P. Van and T.S. Biro, First order and stable relativistic dissipative hydrodynamics, Phys. Lett. B 709 (2012) 106 [arXiv:1109. 0985] [INSPIRE].

[49] P. Kovtun, First-order relativistic hydrodynamics is stable, JHEP 10 (2019) 034 [arXiv: 1907.08191] [INSPIRE].

[50] F.S. Bemfica, M.M. Disconzi and J. Noronha, Nonlinear causality of general first-order relativistic viscous hydrodynamics, Phys. Rev. D 100 (2019) 104020 [arXiv:1907.12695] [INSPIRE]. 
[51] A. Das, W. Florkowski, J. Noronha and R. Ryblewski, Equivalence between first-order causal and stable hydrodynamics and Israel-Stewart theory for boost-invariant systems with a constant relaxation time, Phys. Lett. B 806 (2020) 135525 [arXiv:2001.07983] [INSPIRE].

[52] F. Taghinavaz, Causality and stability conditions of a conformal charged fluid, JHEP 08 (2020) 119 [arXiv : 2004.01897] [InSPIRE].

[53] R.E. Hoult and P. Kovtun, Stable and causal relativistic Navier-Stokes equations, JHEP 06 (2020) 067 [arXiv : 2004.04102] [InSPIRE].

[54] F.S. Bemfica, M.M. Disconzi and J. Noronha, Causality and existence of solutions of relativistic viscous fluid dynamics with gravity, Phys. Rev. D 98 (2018) 104064 [arXiv: 1708.06255] [INSPIRE].

[55] B. Betz, D. Henkel and D.H. Rischke, From kinetic theory to dissipative fluid dynamics, Prog. Part. Nucl. Phys. 62 (2009) 556 [arXiv:0812.1440] [inSPIRE].

[56] G.S. Denicol, T. Koide and D.H. Rischke, Dissipative relativistic fluid dynamics: a new way to derive the equations of motion from kinetic theory, Phys. Rev. Lett. 105 (2010) 162501 [arXiv: 1004.5013] [INSPIRE].

[57] A. Muronga, Relativistic dynamics of non-ideal fluids: viscous and heat-conducting fluids. II. Transport properties and microscopic description of relativistic nuclear matter, Phys. Rev. C 76 (2007) 014910 [nucl-th/0611091] [INSPIRE].

[58] G.S. Denicol, H. Niemi, E. Molnar and D.H. Rischke, Derivation of transient relativistic fluid dynamics from the Boltzmann equation, Phys. Rev. D 85 (2012) 114047 [Erratum ibid. 91 (2015) 039902] [arXiv: 1202.4551] [INSPIRE].

[59] G.S. Denicol, E. Molnár, H. Niemi and D.H. Rischke, Derivation of fluid dynamics from kinetic theory with the 14-moment approximation, Eur. Phys. J. A 48 (2012) 170 [arXiv: 1206.1554] [INSPIRE].

[60] B. Betz, D. Henkel and D.H. Rischke, Complete second-order dissipative fluid dynamics, J. Phys. G 36 (2009) 064029 [INSPIRE].

[61] M.A. York and G.D. Moore, Second order hydrodynamic coefficients from kinetic theory, Phys. Rev. D 79 (2009) 054011 [arXiv:0811.0729] [INSPIRE].

[62] A. Jaiswal, R.S. Bhalerao and S. Pal, New relativistic dissipative fluid dynamics from kinetic theory, Phys. Lett. B 720 (2013) 347 [arXiv: 1204.3779] [INSPIRE].

[63] A. Jaiswal, Relativistic dissipative hydrodynamics from kinetic theory with relaxation time approximation, Phys. Rev. C 87 (2013) 051901 [arXiv:1302.6311] [INSPIRE].

[64] W. Florkowski and R. Ryblewski, Highly-anisotropic and strongly-dissipative hydrodynamics for early stages of relativistic heavy-ion collisions, Phys. Rev. C 83 (2011) 034907 [arXiv: 1007.0130] [INSPIRE].

[65] G.S. Denicol, E. Molnár, H. Niemi and D.H. Rischke, Resistive dissipative magnetohydrodynamics from the Boltzmann-Vlasov equation, Phys. Rev. D 99 (2019) 056017 [arXiv: 1902.01699] [INSPIRE].

[66] R. Biswas, A. Dash, N. Haque, S. Pu and V. Roy, Causality and stability in relativistic viscous non-resistive magneto-fluid dynamics, JHEP 10 (2020) 171 [arXiv:2007.05431] [INSPIRE]. 
[67] D. Kharzeev, Parity violation in hot QCD: why it can happen, and how to look for it, Phys. Lett. B 633 (2006) 260 [hep-ph/0406125] [INSPIRE].

[68] K. Fukushima, D.E. Kharzeev and H.J. Warringa, The chiral magnetic effect, Phys. Rev. D 78 (2008) 074033 [arXiv:0808.3382] [INSPIRE].

[69] D.T. Son and A.R. Zhitnitsky, Quantum anomalies in dense matter, Phys. Rev. D 70 (2004) 074018 [hep-ph/0405216] [INSPIRE].

[70] S. Pu, S.-Y. Wu and D.-L. Yang, Chiral Hall effect and chiral electric waves, Phys. Rev. D 91 (2015) 025011 [arXiv: 1407.3168] [INSPIRE].

[71] N. Banerjee, J. Bhattacharya, S. Bhattacharyya, S. Dutta, R. Loganayagam and P. Surowka, Hydrodynamics from charged black branes, JHEP 01 (2011) 094 [arXiv:0809.2596] [INSPIRE].

[72] M.A. Stephanov and Y. Yin, Chiral kinetic theory, Phys. Rev. Lett. 109 (2012) 162001 [arXiv: 1207.0747] [INSPIRE].

[73] J.-Y. Chen, D.T. Son, M.A. Stephanov, H.-U. Yee and Y. Yin, Lorentz invariance in chiral kinetic theory, Phys. Rev. Lett. 113 (2014) 182302 [arXiv:1404.5963] [INSPIRE].

[74] Y. Hidaka, S. Pu and D.-L. Yang, Relativistic chiral kinetic theory from quantum field theories, Phys. Rev. D 95 (2017) 091901 [arXiv: 1612.04630] [InSPIRE].

[75] A. Lichnerowicz and S.C. for Advanced Studies, Relativistic hydrodynamics and magnetohydrodynamics, Mathematical physics monograph series, W.A. Benjamin, U.S.A. (1967).

[76] A.M. Anile, Relativistic fluids and magneto-fluids, Cambridge University Press, Cambridge, U.K. (2005).

[77] K. Thorne and R. Blandford, Modern classical physics, Princeton University Press, Princeton, NJ, U.S.A. (2017).

[78] J. Anderson and H. Witting, A relativistic relaxation-time model for the Boltzmann equation, Physica 74 (1974) 466.

[79] M.P. Heller, R.A. Janik and P. Witaszczyk, Hydrodynamic gradient expansion in gauge theory plasmas, Phys. Rev. Lett. 110 (2013) 211602 [arXiv: 1302.0697] [INSPIRE].

[80] S. Hess, Tensors for physics, Springer International Publishing, Switzerland (2015).

[81] A. Dash, S. Samanta, J. Dey, U. Gangopadhyaya, S. Ghosh and V. Roy, Anisotropic transport properties of a hadron resonance gas in a magnetic field, Phys. Rev. D 102 (2020) 016016 [arXiv: 2002.08781] [INSPIRE].

[82] P. Mohanty, A. Dash and V. Roy, One particle distribution function and shear viscosity in magnetic field: a relaxation time approach, Eur. Phys. J. A 55 (2019) 35 [arXiv: 1804.01788] [INSPIRE].

[83] A.K. Panda, A. Dash, R. Biswas and V. Roy, Relativistic resistive magneto-hydrodynamics from kinetic theory, in preparation. 